\title{
Broadband Light Generation in Raman-active Crystals Driven by Femtosecond Laser Fields
}

\author{
Miaochan Zhi, Xi Wang and Alexei V. Sokolov \\ Texas A\&M University \\ U. S. A
}

\section{Introduction}

Short pulse generation requires a wide phase-locked spectrum. Earlier the short pulses were obtained by expanding the spectrum of a mode locked laser from self phase modulation (SPM) in an optical fiber and then compensating for group velocity dispersion (GVD) by diffraction grating and prism pairs. Pulses as short as $4.4 \mathrm{fs}$ have been generated (Steinmeyer et al., 1999). For ultrafast measurements on the time scale of electronic motion, generation of subfemtosecond pulses is needed. Generation of subfemtosecond pulses with a spectrum centered around the visible region is even more desirable, due to the fact that the pulse duration will be shorter than the optical period and will allow sub-cycle field shaping. As a result, a direct and precise control of electron trajectories in photoionization and highorder harmonic generation will become possible. But to break the few-fs barrier new approaches are needed.

In recent past, broadband collinear Raman generation in molecular gases has been used to produce mutually coherent equidistant frequency sidebands spanning several octaves of optical bandwidths (Sokolov \& Harris, 2003). It has been argued that these sidebands can be used to synthesize optical pulses as short as a fraction of a fs (Sokolov et al., 2005). The Raman technique relied on adiabatic preparation of near-maximal molecular coherence by driving the molecular transition slightly off resonance so that a single molecular superposition state is excited. Molecular motion, in turn, modulates the driving laser frequencies and a very broad spectrum is generated, hence the term for this process "molecular modulation". By phase locking, a pulse train with a time interval of the inverse of the Raman shift frequency is generated. While at present isolated attosecond X-ray pulses are obtained by high harmonic generation (HHG) (Kienberger et al., 2004), the pulses are difficult to control because of intrinsic problems of X-ray optics. Besides, the conversion efficiency into these pulses is very low (typically $10^{-5}$ ). On the other hand, the Raman technique shows promise for highly efficient production of such ultrashort pulses in the near-visible spectral region, where such pulses inevitably express single-cycle nature and may allow non-sinusoidal field synthesis (Sokolov et al., 2005).

In the Raman technique ns pulses are applied for preparing maximal coherence when gas is used as a Raman medium. When the pulse duration is shorter than the dephasing time $T_{2}$

Source: Advances in Lasers and Electro Optics, Book edited by: Nelson Costa and Adolfo Cartaxo,

ISBN 978-953-307-088-9, pp. 838, April 2010, INTECH, Croatia, downloaded from SCIYO.COM 
$\left(T_{2}=\frac{1}{\pi c \Delta v_{R}}, \Delta v_{R}\right.$ is the Raman linewidth), the response of the medium is a highly transient process, i.e. the Raman polarization of the medium doesn't reach a steady state within the duration of the pump pulse. In this transient stimulated Raman scattering (SRS) regime, a large coherent molecular response is excited. The advantage of using a short pulse is that the number of pulses in the train will be reduced compared with the ns Raman technique. But when a single fs pump is used, the strong SPM suppresses the Raman generation (Kawano et al., 1998). When the pulse duration is reduced to less than a single period of molecular vibration or rotation, an impulsive SRS regime is reached (Korn et al., 1998). In this regime, an intense fs pulse with a duration shorter than the molecular vibrational period prepares the vibrationally excited state and a second, relatively weak, delayed pulse propagates in the excited medium in the linear regime and experiences scattering due to the modulation of its refractive index by molecular vibrations, which results in the generation of the Stokes and anti-Stokes sidebands (Nazarkin et al., 1999). This technique has the advantage of eliminating the parasitic nonlinear processes since they are confined only within the pump pulse duration.

A closely related approach, which is called four-wave Raman mixing (FWRM) for generating ultrashort pulses using two-color stimulated Raman effect, is proposed by Imasaka (Yoshikawa \& Imasaka, 1993). It is based on an experimental result his student has stumbled on. Shuichi Kawasaki was trying to develop a tunable source for thermal lens spectroscopy and he noticed bright, multicolored spots out of the Raman cell pressurized with hydrogen, which they called "Rainbow Star" (Katzman, 2001). The applied beam was supposed to be monochromatic but it actually had two colors in it. To confirm the FWRM hypothesis, a nonlinear optical phenomenon in which three photons interact to produce a fourth photon, they used two-color laser beams with frequencies separated by one of the rotational level splitting for hydrogen $\left(590 \mathrm{~cm}^{-1}\right)$. Indeed, they observed the generation of more than 40 colors through the FWRM process, which provided a coherent beam consisting of equidistant frequencies covering more than thousandths $\mathrm{cm}^{-1}$ in frequency domain (Imasaka et al., 1989). This FWRM process resulted in the generation of higher-order rotational sidebands at reduced pump intensity compared to the stimulated Raman scattering. The generation of the FWRM fields required phase matching and were coherently phased, and therefore had the potential to be used to generate sub-fs pulses (Kawano et al., 1999).

Later ps pulses (Kawano et al., 1996), ps and fs pump pulses (Kawano et al., 1997), and a single fs pulse (Kawano et al., 1998) were used to find the optimal experimental conditions for efficient generation of high-order rotational lines. Generally speaking, when the additional Stokes field is supplied rather than grown from quantum noise, advantages include: highorder anti-Stokes generation, higher conversion efficiency, and improved reproducibility of the pulses generated, as shown in earlier experiments with gas in ns regime (Gathen et al., 1990). Recently, efficient generation of high-order anti-Stokes Raman sidebands in a highly transient regime is also observed using a pair of 100 -fs laser pulses tuned to Raman resonance with vibrational transitions in methane or hydrogen (Sali et al., 2004; 2005). They found that in this transient regime, the two-color set-up permits much higher conversion efficiency, broader generated bandwidth, and suppression of the competing SPM. The high conversion efficiency observed proves the preparation of substantial coherence in the system although the prepared coherence in the medium cannot be near maximal as in the case of the adiabatic Raman technique. 
Almost all these works were carried out using a simple-molecule gas medium such as $\mathrm{H}_{2}$, $\mathrm{D}_{2}, \mathrm{SF}_{6}$ or methane since the gas has negligible dispersion and long coherence lifetime. Molecular gas also has a few other advantages as a Raman medium. They are easily obtainable with a high degree of optical homogeneity and have high frequency vibrational modes with small spectral broadening, which leads to large Raman frequency shifts and large Raman scattering cross sections. However, a Raman gas cell with long interaction length is needed due to the lower particle concentration (Basiev \& Powell, 1999).

What about a solid Raman medium such as a Raman crystal? As we know, the high density of solids results in the high Raman gain. The higher peak Raman cross sections in crystals result in lower SRS thresholds, higher Raman gain, and greater Raman conversion efficiency (Basiev \& Powell, 1999). In addition, there is no need for cumbersome vacuum systems when working with room temperature crystals, and therefore a compact system can be designed.

The difficulty in using crystals is the phase matching between the sidebands because the dispersion in solids is significant. Sideband generation using strongly driven Raman coherence in solid hydrogen is reported but the generation process is very close to that of $\mathrm{H}_{2}$ gas and solid hydrogen is a very exotic material (Liang et al., 2000). Observation of generation with few sidebands (Stokes or anti-Stokes) in other solid material is nothing new. About two decades ago, Dyson et. al. has observed one Stokes (S) and one anti-Stokes (AS) generated on quartz during an experiment designed for another purpose (Dyson et al., 1989). Later, there were numerous works of using Raman crystals for building Raman lasers which extended the spectral coverage of solid-state lasers by using SRS (Pask, 2003). A detailed review of crystalline and fiber Raman lasers is given by Basiev (Basiev et al., 2003). The focus of our work is efficient generation over a broad spectral range. Compared to the crystals that are used for building Raman lasers, the sample we use is much thinner (about 1 $\mathrm{mm}$ or less). The phenomenon that we use in our work is essentially different from SRS: in our regime the generation process is fully coherent, does not depend on seeding by spontaneous scattering, and occurs on a time scale much shorter than the inverse Raman linewidth. We use two-color pumping (with the frequency difference matching the Raman frequency), so our sideband generation is more similar to multiple-order coherent AntiStokes Raman Scattering (CARS) than to SRS.

Therefore this chapter is focused on the development of efficient broadband generation using Raman crystals. Since coherence lifetime in a solid is typically shorter than in a gas, the use of fs (or possibly ps) pulses is inevitable when working with room-temperature solids. We studied broadband sideband generation in a Raman-active crystal lead tungstate $\left(\mathrm{PbWO}_{4}\right)$ either with two $50 \mathrm{fs}$ pulses or a pair of time-delayed chirped pulses (Zhi \& Sokolov, 2007; 2008). Similar broadband generation is also observed in diamond (Zhi et al., 2008). Coherent high-order anti-Stokes scattering has also been observed in many other types of crystals such as $\mathrm{YFeO}_{3}, \mathrm{KTaO}_{3}, \mathrm{KNbO}_{3}$ and $\mathrm{TiO}_{2}$ when two-color femtosecond (fs) pulses are used (Takahashi, 2004; Matsubabra et al., 2006; Matsuki et al., 2007). Great progress has been made recently toward synthesis of ultrashort, even few-cycle pulses using Raman crystal. For example, last year Matsubara et al. have demonstrated promising Fourier synthesizer using multiple CARS signals obtained in a $\mathrm{LiNbO}_{3}$ crystal at room temperature, and generated isolated pulses with $25 \mathrm{fs}$ duration at $1 \mathrm{kHz}$ repetition rate (Matsubara et al., 2008). Very recently, they just realized the generation of pulses in the $10 \mathrm{fs}$ regime using multicolor Raman sidebands in $\mathrm{KTaO}_{3}$ (Matsubara et al., 2009). 
One related technique is the short pulse generation through cascade four-wave mixing (CFWM) of femtosecond pulses in bulk isotropic nonresonant media. Crespo et al. were the first ones to observe the cascade of highly nondegenerate FWM in transparent isotropic condensed medium (150 $\mu \mathrm{m}$ thick BK7 glass slide) and find that the process also corresponds to a coherent scattering effect with geometrically minimized phase mismatch (Crespo et al., 2000). Cascaded FWM and multicolored array generation in a sapphire plate was recently reported by Liu et al., who used two crossing beams of a femtosecond laser (Liu \& Kobayashi, 2008). Cascaded nondegenerate FWM technique for high-power single-cycle pulse synthesis in the visible and ultraviolet ranges was realized very recently by Weigand et al. (Weigand et al., 2009). They generated coherent spectra spanning over two octaves in bandwidths using a thin fused silica slide, which supports a near-single-cycle $2.2 \mathrm{fs}$ visibleUV pulses. The advantage of this method is that tunable multicolor femtosecond pulses can be generated by changing the crossing angle between the two input beams (Liu et al., 2009). Recently, sub-20- fs multicolor laser pulses using cascaded four-wave mixing with chirped incident pulses has been generated in a $1 \mathrm{~mm}$ thick fused-silica glass (Liu \& Kobayashi, 2009). The generation of sub-fs pulse using a solid medium seems very promising.

In this chapter, we will first describe our experimental work on sideband generation in lead tungstate and diamond driven by two color ultrashort fs pulses. We will explain, for example, how the generation is affected by the angle between the pump beams, the detuning between the two pump frequencies, the polarization, etc. We will describe how we achieve efficient sideband generation by using a pair of time delayed linearly chirped pulses. After that, we will discuss the two dimensional colored array generation in lead tungstate and diamond when three femtosecond pulses are focused in the crystals. We will show some interesting unsolved "mysterious" experimental results we encountered in our experiment, which may inspire theoretical simulations. At last, we will propose future work that can be extended from this research.

\section{Experiment setup}

The schematics of the setup is shown in Fig. 1 (a). The seed laser (Mira, Coherent) is a modelocked (Kerr Lens Mode-locking) ultrafast laser that produces ultrashort, wide bandwidth $(>50 \mathrm{~nm})$, fs pulses ( $<20 \mathrm{fs})$ using Ti:Sapphire as the gain medium. The output pulse has a repetition rate of $76 \mathrm{MHz}$ with a wavelength at $800 \mathrm{~nm}$. The pulses are used to seed an amplifier (Legend, Coherent). The pulse is first stretched by a grating to about 200 ps and then is amplified by a Ti:Sapphire regenerative amplifier system pumped by a pulsed, frequency doubled Nd:YLF laser at $523.5 \mathrm{~nm}$. It is compressed afterwards by the grating compressor to a pulse with energy $>1.0 \mathrm{~mJ}$ at a $1 \mathrm{kHz}$ repetition rate and pulse duration of $<35 \mathrm{fs}$ FWHM. The energy stability is $<1 \%$ RMS. This amplified pulse is used to pump two computer-controlled optical parametric amplifiers (OPerA, Coherent). It is first down-converted to a signal pulse (the short wavelength, 1150-1600 nm) and an idler pulse (the long wavelength, 1600-2630 $\mathrm{nm}$ ) by a parametric process. These pulses obtained from the two OPAs can be frequency doubled or mixed with the fundamental pulses to produce up to $30 \mu \mathrm{J}$ per $50 \mathrm{fs}$ Gaussian pulse at tunable visible wavelengths. For example, the SHG of the signal beam will produce pulses with wavelength ranging from 575 to $800 \mathrm{~nm}$. The sum frequency generation (SFG) will produce pulses with wavelength range from 533 to $613 \mathrm{~nm}$. These pulses are commonly used in our experiment. With the fundamental pulse, we can choose three pulses to use for excitation. For the convenience of description, here we follow 


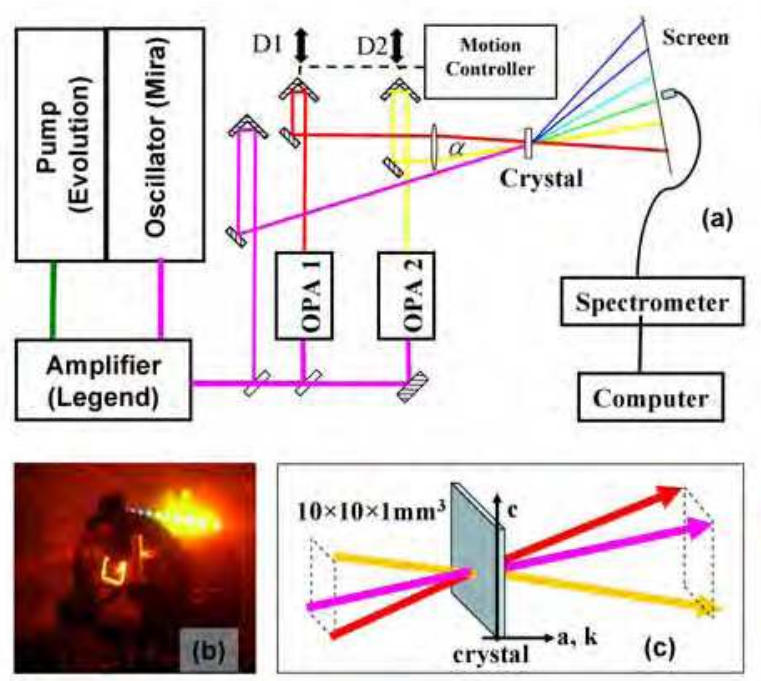

Fig. 1. (a) Experimental setup. D1, D2 are retro-reflectors. They are mounted on a motorcontrolled translation stage. OPA: Optical Parametric Amplifier. The spectra are measured with an Ocean Optics fiber-coupled spectrometer. (b) A picture of the mounted crystal $\left(\mathrm{PbWO}_{4}\right)$ and the generated sidebands which are projected to a screen behind the crystal. (c) A schematics of the beam geometry with respect to the crystal axises.

the CARS terminology. For the first two pulses, we call the short wavelength one as pump and the longer one as Stokes.We call the third pulses as probe. Normally a small fraction of the fundamental pump pulse (fixed wavelength) is used as the Stokes pulse while the output from the two OPAs (tunable) are either served as a pump or probe pulse. We typically use 1 to $2 \mu \mathrm{J}$ per pulse focused to about a $100 \mu \mathrm{m}$ size spot at the sample. This laser intensity is right below the onset of (strong) SPM. The laser beams are typically sent perpendicular to the large surface and crossed at the crystal after the focal lens with a small angle $a$, which varies from 2 to 7 degrees. The retro-reflectors in one or two beams (if a probe beam is used) are mounted on a motor controlled translation stage so that the delay between the pulses can be varied with a precision of $1 \mu \mathrm{m}$, which is about $6.7 \mathrm{fs}$. The spectra are measured with a fiber-coupled spectrometer (Ocean Optics, USB 2000, slit size $25 \mu \mathrm{m}$, measures wavelength ranging from 180 to $870 \mathrm{~nm}$ ), which has an average optical resolution of $1.4 \mathrm{~nm}$, with a slightly higher resolution in the visible region. We take the pictures of the sidebands projected on a piece of white paper. In Fig. 1 (b) we show a picture of the crystal together with the sidebands generated and projected on a piece of paper. In Fig. 1 (c) we show a typical geometry of the input beams.

\section{Broadband light generation in Raman crystals with two-color laser fields}

\subsection{Broadband light generation in $\mathrm{PbWO}_{4}$ driven by two fs pulses (Zhi \& Sokolov, 2007)}

The first sample we choose is $\mathrm{PbWO}_{4}$ (lead tungstate), which exhibits good optical transparency (from 0.33 to $5.5 \mu \mathrm{m}$ ), high damage threshold, and is non-hygroscopic. It is also 
a popular crystal used for building Raman lasers using ns or ps pulsed pumping (Kaminskii et al., 2000). The $\mathrm{PbWO}_{4}$ sample has a size of $10 \times 10 \times 1 \mathrm{~mm}^{3}$, with the large surface perpendicular to the a-axis of the crystal. $\mathrm{PbWO}_{4}$ has a strong narrow Raman line at $901 \mathrm{~cm}^{-1}$ with linewidth $\Delta v_{R}=4.3 \mathrm{~cm}^{-1}$, which corresponds to a phonon relaxation time $T_{2} \approx 2.5$ ps. In the past, when much longer single-color 100 ps pulses were applied to $\mathrm{PbWO}_{4}$ (steady state regime, pulse duration $\tau_{p}>T_{2}$ ), several high-order Stokes and anti-Stokes sidebands were generated (Kaminskii et al., 2000). Another relatively strong Raman line at $325 \mathrm{~cm}^{-1}$ has a linewidth of $7.5 \mathrm{~cm}^{-1}$, which is almost comparable to the width of $901 \mathrm{~cm}^{-1}$ line for a certain crystal orientation.

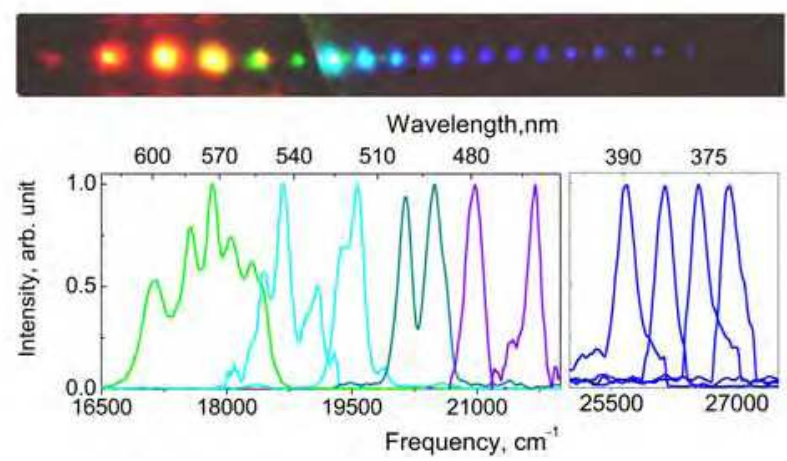

Fig. 2. Broadband generation in $\mathrm{PbWO}_{4}$ crystal with two pulses (at $588 \mathrm{~nm}$ and $620 \mathrm{~nm}$ ) applied at an angle of 4 degrees to each other. Top: generated colors projected on a white paper screen. The two input pulses (bright yellow and red spots), two $S$ and two AS are attenuated by an neutral-density filter. Note that the line connecting the color spots has a slight cusp at intermediate AS orders. Bottom: spectra of the generated sidebands (left: AS 1 to AS6; right: AS 12 to AS 16). The frequency spacing between the sidebands at higher orders decreases gradually.

Using two-color ultrashort pulses (transient regime, $\tau_{p}>>T_{2}$ ), we observe efficient generation of many sidebands in $\mathrm{PbWO}_{4}$ when two pulses $\left(\lambda_{1}=620 \mathrm{~nm}, \lambda_{2}=588 \mathrm{~nm}, \delta \omega=930\right.$ $\mathrm{cm}^{-1}$ ) with parallel polarizations are crossed at the crystal with an angle of 4 degrees (Fig. 2). The angles are measured external of the crystal except stated otherwise. The sidebands emerge spatially well-separated and have the same polarization as the two input beams. Up to 20 AS and $2 \mathrm{~S}$ sidebands are observed on a white paper screen which is put about $25 \mathrm{~cm}$ away from the crystal (Fig. 2 top).

The spectrum of the first 6 AS and the higher order sidebands (AS 12 to AS 16) is shown in Fig. 2 (bottom). Note here we call the frequency upshifted sideband as anti-Stokes (AS) and the frequency downshifted ones as Stokes (S). The spectra of the lower-order sidebands show a rich structure, due to simultaneous excitation of several Raman lines by the large spectral width of the fs laser pulses. The frequency spacing between the sidebands decreases gradually and reaches about $450 \mathrm{~cm}^{-1}$ at the highest orders measured, to our surprise.

To prove the Raman-resonant nature of sideband generation, and to separate the effect of instantaneous FWM, we tune the difference between the two applied laser frequencies $(\delta v)$, and measure the generated AS frequencies. Fig. 3 shows these generated frequencies as a function of the angle at which sidebands emerge from the $\mathrm{PbWO}_{4}$ crystal. We perform this 
measurement at a relatively large input beam crossing angle of 6 degrees. At this angle, and at sufficiently large $\delta v\left(1804\right.$ or $\left.2002 \mathrm{~cm}^{-1}\right)$, the generated AS 1 beam splits into two slightly separated distinctively colored beams: one corresponding to (non-resonant) FWM, and the other (which is much brighter) corresponding to Raman-resonant AS generation. By moving the fiber tip of the spectrometer to the location shown in Fig. 3 (insert) by an arrow, we measure the FWM frequency (as opposed to the Raman-shifted frequency measured at the center of the main AS 1 beam). We observe that as we vary $\delta v$ from 844 to $2002 \mathrm{~cm}^{-1}$, the Raman sidebands are generated at approximately the same angle and with roughly the same frequency shift from the previous order, while the FWM frequency varies as $v_{F W M}=2 v_{2}-v_{1}$. The observed decrease in the frequency spacing between higher-order sidebands is likely due to phase matching.

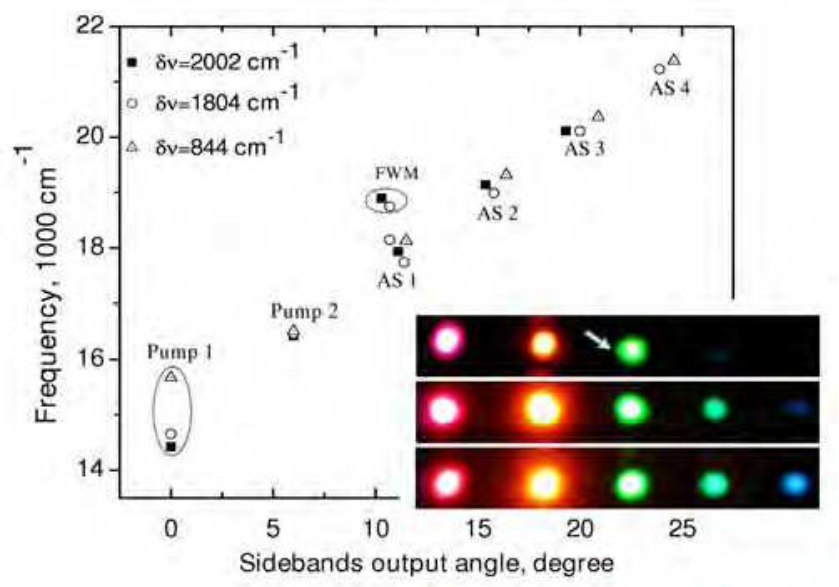

Fig. 3. Peak frequency of the generated sidebands, plotted as a function of the output angle. One input frequency (pump 2) is fixed while the $\delta v=v_{2}-v_{1}$ is tuned to $844 \mathrm{~cm}^{-1}$ (triangles), $1804 \mathrm{~cm}^{-1}$ (circles), and $2002 \mathrm{~cm}^{-1}$ (squares) respectively. The FWM frequency (measured at the point shown on the insert by the arrow) varies as $v_{F W M}=2 v_{2}-v_{1}$ while the Raman sideband frequencies stay approximately fixed. The insert shows the output beams projected onto a screen, for these same values of $\delta v$ (varying from 2002 to $844 \mathrm{~cm}^{-1}$ top to bottom).

When we vary the angle between the two applied laser beams (while keeping the two wavelengths fixed), we observe substantial changes in both the AS frequency shifts, and in the conversion efficiency. AS conversion is negligible for collinear input beams. The optimum conversion in $\mathrm{PbWO}_{4}\left(\right.$ for $\delta v=930 \mathrm{~cm}^{-1}$ ) is achieved when the angle between the applied beams is $4^{\circ}$. When the angle is further increased, AS conversion decreases, while the frequency separation of the AS sidebands goes up. Apparently, phase matching plays a critical role in the generation of multiple spectral sidebands in Raman-active crystals (as compared to the collinear Raman generation in gasses (Sokolov \& Harris, 2003; Sokolov et al., 2005)). Even though at larger beam crossing angles the conversion efficiency is expected to decrease because of the reduced beam overlap, for angles below $7^{\circ}$ it is the phase matching, along with the spectrum of exited Raman transitions, that determines the conversion efficiency and the frequencies generated in thin crystals. 
The generation is very sensitive to the polarization of the beams. First of all, it has been shown that the Raman gain is strongly peaked when the crystal is excited by a beam whose polarization is parallel to the c-axis of the crystal in the SRS experiment (Kaminskii et al., 2000). Secondly, we find that the generation is best when the pump and Stokes beams have the parallel polarization, which is either parallel or normal to the c-axis. Thirdly, the generation has different frequency spacing when the polarization of the pump beams are parallel with different axis of the crystal as shown in Fig. 4. When we change the polarization of the input beams from $S$ (the polarization is parallel to the plane containing the optical axis $\mathrm{c}$ and the wavevector k, see Fig. 4 (a)) to P (perpendicular to S), we observe a significant variation in the generated frequency spacing. We speculate that this is the result of dispersion since when $\mathrm{P}$ polarization is used, the crystal sees refractive index of $\mathrm{n}_{o}$ (larger than $\mathrm{n}_{e}$ ).
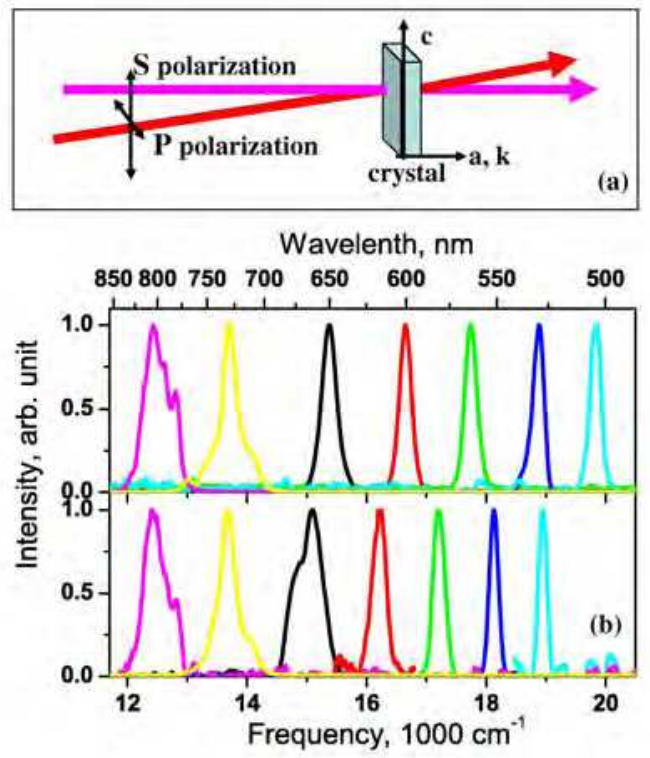

Fig. 4. (a) Crystal orientation and the two possible polarization of the laser beams. (b) The spectra of the two pump beams and the first 5 AS generated in the $\mathrm{PbWO}_{4}$ crystal when two pulses (at $729 \mathrm{~nm}$ and $805 \mathrm{~nm}$ ) are applied at two different polarizations [P (top) and S (bottom)]. The sideband frequencies have different frequency spacings.

\subsection{Broadband light generation in diamond driven by two fs pulses (Zhi et al., 2008)}

The motivation to use diamond for broadband generation is two-fold. Firstly, diamond has a single strong narrow Raman line at a very large frequency shift $\left(1332 \mathrm{~cm}^{-1}\right)$, compared to the other crystals (Basiev et al., 1999). Secondly, diamond is isotropic and the refractive index is well known, which makes it easier (compared to $\mathrm{PbWO}_{4}$ we studied) to do some theoretical calculation and thus help us understand the complicated experimental results.

Beside the above-mentioned two reasons, diamond also has several remarkable properties which are desirable for broadband generation (Pierson, 1993). First of all, diamond is capable of transmitting over an unusually broad spectral range (from X-ray region to the microwave and $\mathrm{mm}$ wavelengths) and has the widest electromagnetic bandpass of any 
material. This broadband transmission is essential for the sideband generation. Second, it has an extremely high thermal conductivity (five times that of copper) and is extremely chemically inert. Therefore, it is not easy to get damaged by a laser and it does not require much protection from moisture. At last, diamond has the highest atom density of any material. A high density means a high Raman gain.

Back in 1963, diamond was chosen as a representative covalent crystal for the SRS experiment, one AS and two $\mathrm{S}$ were observed when a high intensity $\left(20 \mathrm{MW} / \mathrm{cm}^{2}\right)$ Ruby laser was used for excitation (Eckhardt et al., 1963). Natural diamonds are costly and scarce. Recently, synthesis of large area diamonds at lower pressure using chemical vapor deposition (CVD) technique was developed. Kaminskii et al. used CVD diamond for Raman laser converter based on SRS and reported observation of SRS (up to $1 \mathrm{~S}$ and 3 AS sidebands) in diamond (using both ns and ps pulses) (Kaminskii et al., 2005; 2006).

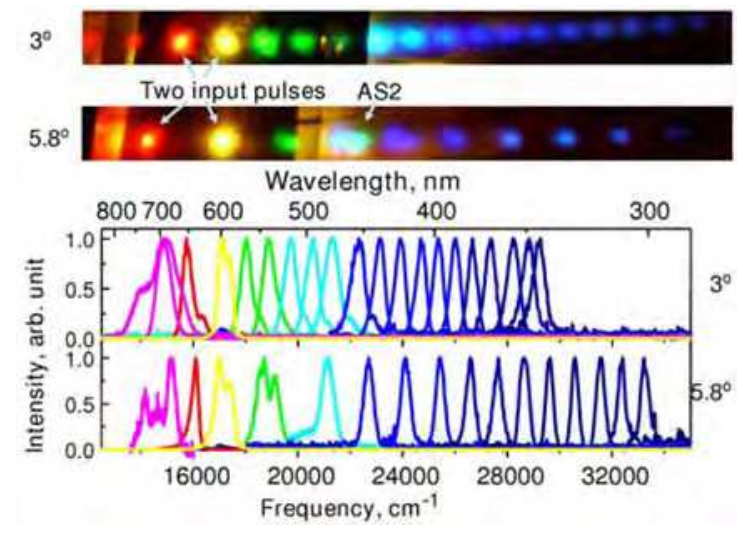

Fig. 5. Broadband generation in diamond with two input pulses $\left(\lambda_{1}=630 \mathrm{~nm}\right.$ and $\lambda_{2}=584$ $\mathrm{nm}, \delta v=1250 \mathrm{~cm}^{-1}$ ) crossed at angles of $3^{\circ}$ and 5.8 . Top: Generated beams projected onto a white screen. The two pump beams, S 1 and the first few AS beams are attenuated (after the sample) by a neutral-density filter. The AS 2 spot clearly shows two different colors, with blue corresponding to the Raman generation and green to the FWM signal. Bottom: Normalized spectra of the generated sidebands.

The experimental setup is about the same as the one we used for $\mathrm{PbWO}_{4}$ crystal. We start with an angle $3.6^{\circ}$ (the phase matching angle between the peak wavelengthes) between the pair of beams. By applying two $50 \mathrm{fs}$ pulses (at $\lambda_{1}=630 \mathrm{~nm}, \lambda_{2}=584 \mathrm{~nm}$, and $\delta v=1250 \mathrm{~cm}^{-1}$ ), we obtain generation of up to 16 AS and $2 \mathrm{~S}$ sidebands. The highest frequency generated is in the UV region at a wavelength of $301 \mathrm{~nm}$. Similar to the sideband generation in $\mathrm{PbWO}_{4}$, the sidebands are not equally spaced in frequency. The energy conversion from pump to AS 1 is $3 \%, 2 \%$ to the $\mathrm{S} 1$ and $0.5 \%$ to AS 2 . The total energy conversion from pump to sidebands is $14 \%$. The sidebands generated using $3^{\circ}$ crossing angle overlap substantially in spectrum with each other.

From Fig. 5, we can clearly see that the instantaneous four-wave-mixing (FWM) signal coexists with Raman generation in the lower orders of the sidebands. The AS 2 beam spot shows two colors: blue and green. By measuring the frequency shift from the preceding sideband, we deduce that the blue is due to Raman generation, while the green beam corresponds to the FWM signal. 
We find that sidebands generated at $5.8^{\circ}$ have a larger (about twice) frequency spacing compared to the $3^{\circ}$ case when we plot the sideband frequency versus the sideband order as shown in Fig. 5 top picture. Also the sidebands come out at a much larger angle spacing as shown in the Fig. 5 bottom picture (we define the Stokes output angle as 0 degree). This is more obvious for low-order sidebands which are more affected by the strong FWM signal. This shows that the initial phase matching condition between the two pump beams decides the generated sideband output angles and frequencies.

We keep the pump frequency the same while tuning the Stokes frequency from $820 \mathrm{~cm}^{-1}$ to $2608 \mathrm{~cm}^{-1}$ and record the sideband frequency. The result is shown in Fig. 6. When $\Delta v$ varies from $820 \mathrm{~cm}^{-1}$ to $2608 \mathrm{~cm}^{-1}$, the output angle doesn't vary much. Also, the frequency vary much less compared to the change of $\Delta v$. These facts are clear indications that the signals are generated mainly through the Raman process. This also shows that the tuning to exact Raman resonance is not necessary as we can obtain generation even when $\Delta v$ is twice the Raman frequency. When the phase matching condition is satisfied, the low-order sidebands are very strong because of the boost from the FWM process.

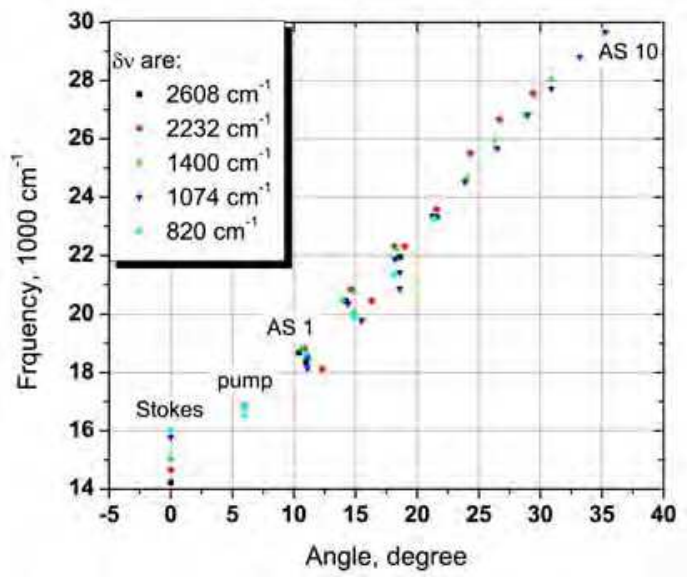

Fig. 6. The Sideband frequency and output angle at the different detuning ( $\Delta v$ vary from 820 $\mathrm{cm}^{-1}$ to $2608 \mathrm{~cm}^{-1}$ ) between the pump (fixed at $594 \mathrm{~nm}$ ) and Stokes pulses. The output angles do not vary much, nor does the frequencies of the sidebands, although $\Delta v$ varies a lot.

Due to the material dispersion, phase matching is optimized when different frequency components propagate at different angles. We perform calculations that support our qualitative understanding of the role of phase matching using a pair of Gaussian pulses, which are very close to those we measure in experiment using a spectrometer. We take the spectral intensity of an n's AS sideband as given by

$$
I_{n}(v) \sim I_{n-1}\left(v-v_{R}\right) \cdot \operatorname{sinc}^{2}\left\{\left[\vec{k}(v)-\vec{k}\left(v-v_{R}\right)-\vec{k}_{R}\right] L / 2\right\} .
$$

Here $\vec{k}$ is the wavevector $(k=n v / c), v_{R}$ is the Raman shift and $c$ is the speed of light. The refractive index of diamond $n$ is calculated by using the generalized Cauchy dispersion formula (Smith et al., 2001):

$$
n(\lambda)=2.37-1.0 \times 10^{-5} /(1.24 / \lambda)^{2}+8.0 \times 10^{-3} \times(1.24 / \lambda)^{2}+1.0 \times 10^{-4} \times(1.24 / \lambda)^{4},
$$


with $\lambda$ in units of $\mu \mathrm{m}$. This formula fits well with the available experimental data (Piersonf, 1993; Edwards \& Ochoa, 1981). The k-vector of the Raman excitation $\vec{k}_{R}$ is determined by the directions of the two input beams. We assume that the angle between the low-order sidebands is approximately the same.

We find that when the angle is smaller than the phase matching angle, the peak frequency shift of the generated AS 1 from the pump pulse is $1163 \mathrm{~cm}^{-1}$, which is smaller than the Raman shift. The opposite happens when an angle larger than the phase matching angle is applied, as shown in Fig. 7. The peak frequency shift of the generated AS 1 from the pump pulse is $1301 \mathrm{~cm}^{-1}$ for input angle of $3.6^{\circ}$ and $1466 \mathrm{~cm}^{-1}$ at $4.6^{\circ}$. This agrees with our experimental observations in diamond. The peak frequencies of the two input pulses phase match at $3.6^{\circ}$. Therefore, when the two input pulses cross at $5.8^{\circ}$, the generated spectrum has a larger frequency spacing than that of the $3^{\circ}$. This calculation may also explain the gradually decreasing frequency spacing between the sidebands, since due to the normal medium dispersion, the optimum beam-crossing angle increases with increasing sideband frequency.

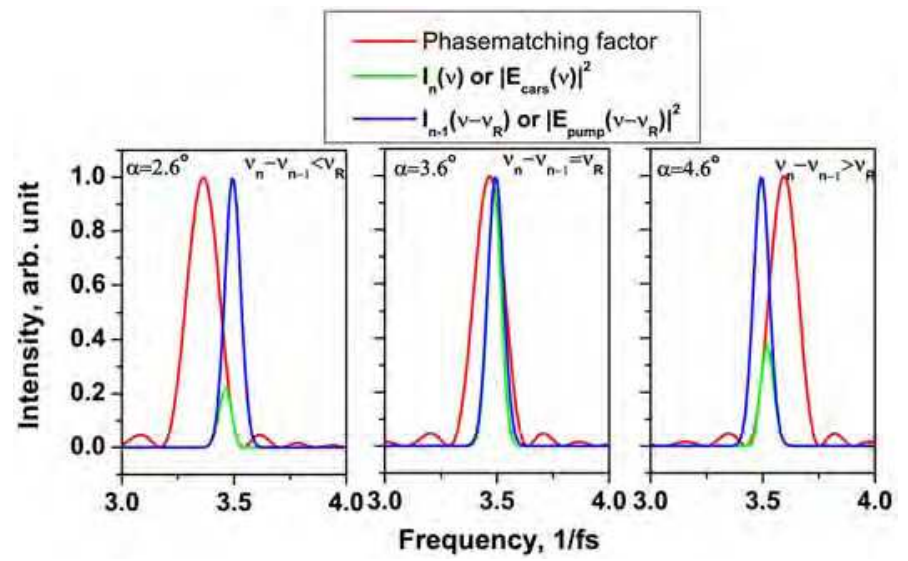

Fig. 7. Theoretical calculation of the generated AS 1 when the two pumps cross at angle 2.6, 3.6 and 4.6 degree respectively, with the 3.6 degree corresponding to the phase matching angle between two input pulses. The input pulses have the central wavelengths of is $630 \mathrm{~nm}$ and $581.23 \mathrm{~nm}$, respectively. The frequency difference is exactly the Raman shift $1332 \mathrm{~cm}^{-1}$. The sample thickness used for calculation is $500 \mu \mathrm{m}$. The phase matching factor is $\operatorname{sinc}^{2}(\delta \mathrm{k}$ $* \mathrm{~L} / 2)$, where $\delta \mathrm{k}$ is the phase mismatch.

\subsection{Broadband light generation in $\mathrm{PbWO}_{4}$ by excitation of the Raman mode at 325 $\mathrm{cm}^{-1}$}

\subsubsection{Excitation with two fs pulses}

With fs pulse pumping, the SRS gain increment explicitly depended on the integral cross section instead of the peak cross section of spontaneous Raman scattering (Basiev et al., 2004) therefore the excitation of $325 \mathrm{~cm}^{-1}$ line can dominate when phase matching is satisfied. When we reduce the angle between the pump and Stokes beams to 2.9 degree and tune the frequency difference below $600 \mathrm{~cm}^{-1}$, we observe generation due to excitation of the Raman mode at $325 \mathrm{~cm}^{-1}$ as shown in Fig. 9 (a). Due to the small phase matching angle 
between the two input pulses, the output beams are very close to each other and become inseparable for high-order sidebands. We see the interplay between the phase matching and Raman resonance when we fix the Stokes pulse at $804 \mathrm{~nm}$ wavelength while tunning the pump pulse from 760 to $780 \mathrm{~nm}$, with a detuning varying from 408 to $615 \mathrm{~cm}^{-1}$. Although it is far from Raman resonance at $615 \mathrm{~cm}^{-1}$ detuning, the phase matching condition between the pump and Stokes is satisfied, which results in generation of 22 AS and 2 S. The phase matching between the pump and probe fields is also good, as can be seen from the FWM signal (blue) shown in the picture. As many as 10 CARS signals are measured, which indicates a significant coherence being built up in the crystal.

Because of the lower frequency spacing between the two pumps and the wide spectral width of the fs pulses, the smaller rotational modes are all excited so that the lower order sidebands have multiple peaks. This is different from the multiple peaks generated when $901 \mathrm{~cm}^{-1}$ transition is excited. Those multiple peaks are due to the coexistence of the FWM and Raman signals. The high-order sidebands are about equally spaced at a $320 \mathrm{~cm}^{-1}$ spacing. The angle between the two applied beams is 2.5 degree, which corresponds to 1.2 degrees inside the crystal. Thus it might be possible to generate sidebands with a collinear configuration.

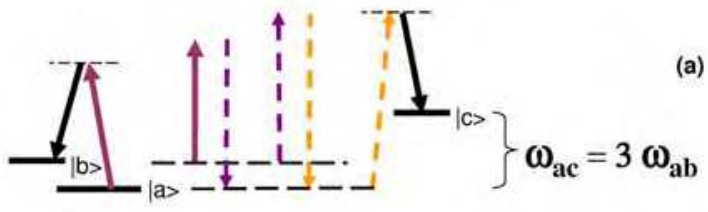

(a)

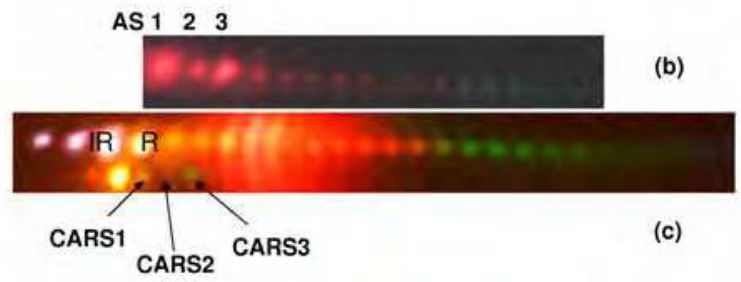

Fig. 8. (a) Energy level schematics; (b) The AS 3 generated by pump and Stokes beams which are tuned to excite the Raman mode at $325 \mathrm{~cm}^{-1}$ is stronger than AS 2 due to the excitation of Raman mode at $903 \mathrm{~cm}^{-1}$; (c) CARS 1, 2 and 3 signals generated by a delayed probe pulse.

One interesting feature we noticed in our experiment is that the high order AS 3 is stronger than AS 2 when the $325 \mathrm{~cm}^{-1}$ is excited with pump pulse at $770 \mathrm{~nm}$ and Stokes pulse at 805 $\mathrm{nm}$, as shown in Fig. 8 (b). What happens can be explained by a schematic energy level diagram as shown in Fig. 8 (a). The Raman mode width in the crystal is larger than the one in a gas. Consequently, although $\omega_{R 1}=903 \mathrm{~cm}^{-1}$ is not exactly three times the $\omega_{R 2}=325 \mathrm{~cm}^{-1}$, it is still possible that the AS 3 gets enhanced by the Raman mode at $903 \mathrm{~cm}^{-1}$. A clear indication is shown in Fig. 8 (c) where a third probe beam is used. The picture is taken with a delayed probe, therefore only CARS signal can survive. The CARS 3 signal, which is generated due to excitation of $901 \mathrm{~cm}^{-1}$, is very strong, compared to the CARS 2 signal.

\subsubsection{Excitation with a pair of time-delayed linear chirped pulses}

In the above experiments we used two nearly transform-limited $50 \mathrm{fs}$ laser pulses tuned such that their frequency difference was approximately equal to the Raman frequency. One 
complication in those experiments was simultaneous excitation of several Raman lines by the large spectral width of the fs laser pulses. Also, the high-peak intensity of the pulses leads to generation of the strong instantaneous FWM signals. These resulted in a rich structure of the lower-order sidebands' spectra. In addition, the total laser energy fluence in those experiments was limited by "parasitic" nonlinear processes, such as self-focusing and SPM, which can strongly distort the pulses. Only 2 to $3 \mathrm{~mW}$ of average beam power can be used.

Femtosecond pulse sequencing and shaping allows selective (narrow-band) Raman excitation by broad-band pulses, as discussed in the past (Zheltikov, 2002; Gershgoren et al., 2003; Dudovich, 2002). At the same time, the shaped pulses are necessarily longer than transform-limited, and therefore have lower peak intensity, helping to avoid the onset of parasitic nonlinear processes at larger energy fluences.

We obtain chirped pulses by a misalignment of the compressor in the amplifier. The pulse is then split into two by an ultrafast beamsplitter and the delay between the two pulses can be varied. The two beams are recombined and focused in the crystal by a 2 inch diameter lens (focal lens $\mathrm{f}=50 \mathrm{~cm}$ ). Both beams are attenuated by variable neutral density filters so that the power used is below the threshold for the parasitic nonlinear processes. The power used ranges from $5 \mathrm{~mW}$ to $20 \mathrm{~mW}$, depending on the pulse chirp and the focusing conditions.

A linearly chirped pulse can be written as:

$$
E(t)=\exp \left(-a t^{2}\right) * \exp \left[I *\left(b t^{2}+\omega_{l} t\right)\right] .
$$

Here $1 / \sqrt{a}$ is roughly the pulse duration, $\mathrm{b}$ is the chirp rate of the pulse, and $\omega_{l}$ is the laser center frequency.

The intensity for two time-delayed linearly chirped pulses is:

$$
\begin{aligned}
I(t) & =\left|E(t)+E\left(t-t_{d}\right)\right|^{2} \\
& =\exp \left(-2 a t^{2}\right)+\exp \left[-2 a\left(t-t_{d}\right)^{2}\right] \\
& +2 \exp \left[-a\left(t^{2}+\left(t-t_{d}\right)^{2}\right] \cos \left[b\left(2 t-t_{d}\right) t_{d}+\omega_{l} t_{d}\right] .\right.
\end{aligned}
$$

When $\Delta \omega=\omega_{R}=b t_{d}$, the last term in $I(t)$ is proportional to $\cos \left(2 \omega_{R} t+C\right)\left[C=\left(\omega_{l} \omega_{R}-\omega_{R}^{2}\right) / b\right.$, is a constant]. We see that there is a periodic beat in the pulse intensity in the time domain. The periodicity of this pulse train can be matched to the period of this Raman mode at $\omega_{R}$, permitting selective mode excitation (Gershgoren et al., 2003). One can adjust the excitation frequency simply by adjusting the time delay between the two pulses. A related method of achieving the spectral selectivity is Fourier domain pulse shaping, where a pulse train is created by applying a periodic spectral phase to a single fs pulse using a pulse shaper (Dudovich, 2002).

For a fixed $\Delta \omega=\omega_{R}, t_{d}$ is proportional to $1 / b$, so the slope of $t_{d} v s$. the inverse of the chirp rate gives the Raman frequency $\omega_{R}$ (which is to be excited). We calculate the chirp rate $b$ from $\mathrm{b}=\omega_{R} / t_{d}$ by measuring the $\mathrm{t}_{d}$ using pulses with different chirp rate when the Raman mode at $325 \mathrm{~cm}^{-1}$ is excited.

The pulse that is used here is negatively chirped, with a pulse duration full width at half maximum (FWHM) ranging from $80 \mathrm{fs}$ to 2 ps. The chirp is approximately linear. The available spectral width (FWHM) of the pulse is around $460 \mathrm{~cm}^{-1}$. The angle between the pump beams is about $2.4^{\circ}$. We observe as many as 40 AS at two different delays. The output angle of the highest-order sideband with respect to the pump beam is about $80^{\circ}$. For convenience of description, we call the pump beam with variable pulse delay as the 
variable-delay pump and the other one as the fixed-delay pump beam. We observe AS generation on the variable-delay pump side at a positive pulse delay (here the variabledelay pump acts as the pump beam and the fixed-delay pump acts as the Stokes beam, as conventionally $\left.\omega_{\text {pump }}>\omega_{\text {Stokes }}\right)$. When we move the pulse forward from 0 delay to $-t_{d}$, we observe AS generation on the side of the fixed-delay pump beam (as expected), since now the fixed-delay beam functions as a pump beam.

When we change the pulse chirp to $620 \mathrm{~cm}^{-1} / \mathrm{ps}$, we observe, unexpectedly, that some of the high-order sidebands are stronger than the low-order ones, as shown in Fig. 9 (d). This can be seen even more clearly when a larger angle between the two pump beams is used and when the Raman mode at $903 \mathrm{~cm}^{-1}$ is excited (as described below). Kuon Inoue et al. have observed a similar non-monotonous variation of spectral intensity of the generated sidebands when using two-color subpicosecond excitation in $\mathrm{TiO}_{2}$ crystal (Inoue et al., 2007). We believe this maybe due to the large phase mismatch of certain sidebands. Another possible explanation is based on our experimental observation. Since the FWM coexists with the Raman process, certain sideband gets enhanced when the two processes overlap at that order.

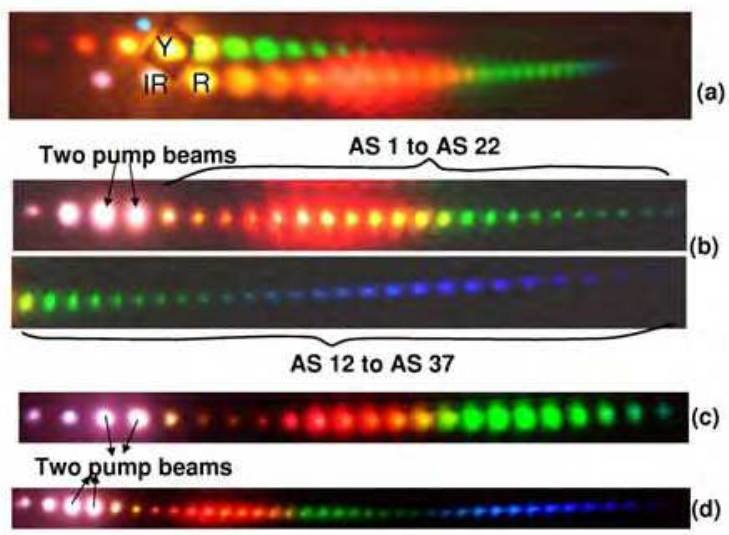

Fig. 9. (a) Broadband generation in $\mathrm{PbWO}_{4}$ pumped by nearly-transform-limited fs pulses $\left(\mathrm{IR}, \lambda_{\text {Stokes }}=804 \mathrm{~nm}, \mathrm{R}, \lambda_{\text {pump }}=766 \mathrm{~nm}\right)$. The angle between the pump and Stokes beams is $29^{\circ}$. A third probe pulse $(\mathrm{Y})$ leads to generation of many orders of $S$ and AS sidebands. (b) and (c) Broadband generation in $\mathrm{PbWO}_{4}$ using two time-delayed linearly chirped pulses applied at angle of $2.4^{\circ}$ to each other. The pulse chirp is about $1280 \mathrm{~cm}^{-1} /$ ps for part (b), and 440 $\mathrm{cm}^{-1} / \mathrm{ps}$ for part (c). (d) The intensity modulation of the generated sidebands is clearly seen when the pulse chirp is about $620 \mathrm{~cm}^{-1} / \mathrm{ps}$.

Broadband generation at a chirp rate of $1280 \mathrm{~cm}^{-1} / \mathrm{ps}$ and a pulse delay of $0.7 \mathrm{ps}$ are shown in Fig. 9 (b). We observe 40 AS and 3 S sidebands. The frequency separation between the sidebands is around $320 \mathrm{~cm}^{-1}$ on average. It decreases to $240 \mathrm{~cm}^{-1}$ for the high-order sidebands. We measure high nonlinear conversion efficiency. As much as $41 \%$ of the pump pulse energy and $21 \%$ of the Stokes pulse energy are converted into the generated sidebands. When the chirp rate is increased to $1060 \mathrm{~cm}^{-1} / \mathrm{ps}$, the sideband generation seems to become less effective. The number of observed AS sidebands decreases to 34 . The conversion efficiency from the pump and Stokes beams decreases to 33\% and 19\%, respectively. When we introduce more chirp until a chirp rate of $440 \mathrm{~cm}^{-1} / \mathrm{ps}$ is reached, the 
efficiency reduces to about $14 \%$ for the pump and $11 \%$ for the Stokes beam. About 22 AS sidebands are observed. However, the substantial pulse chirping, as well as the relatively loose beam focusing, allow us to use a rather high combined average power of over $15 \mathrm{~mW}$ (measured after the crystal) without introducing the parasitic effects. Another feature of the generation using pulses with a high chirp rate is that the FWM signal gets weaker. Consequently, the generated sidebands have good beam profiles, as shown in Fig. 9 (c), and their spectra are mostly single-peaked.

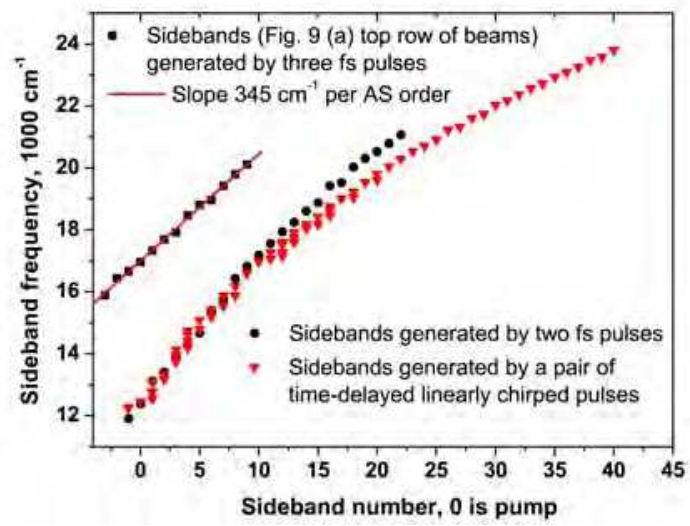

Fig. 10. Comparison of the sideband generation in $\mathrm{PbWO}_{4}$ using two nearly-transformlimited fs pulses $\left(\lambda_{\text {Stokes }}=804 \mathrm{~nm}\right.$ and $\left.\lambda_{\text {pump }}=766 \mathrm{~nm}\right)$ and a pair of time-delayed chirped pulses. Many more sidebands are generated in the latter case.

In Fig. 10 we plot the sideband frequency as a function of the sideband order. The squares show the peak frequencies of the sidebands generated by the pump (at $760 \mathrm{~nm}$ ) and Stokes pulses (at $804 \mathrm{~nm}$ ). The frequency spacing decreases slightly as the order goes higher. We also plot the many orders of CARS signals generated by all three pulses. We can see that the CARS signal has a regular frequency spacing of $345 \mathrm{~cm}^{-1}$, which is close to the spontaneous Raman frequency measured at $325 \mathrm{~cm}^{-1}$. The probe beam (Yellow) is labeled as 0 order. Compared to the sidebands generated by two (nearly-transform-limited) fs pulses (Fig. 10, filled circles), there are many more sidebands generated when a pair of time delayed linearly chirped pulses are used (Fig. 10, triangles). The AS sidebands span a range of $12,000 \mathrm{~cm}^{-1}$.

\section{Coherence between the generated sidebands (Zhi \& Sokolov, 2007)}

We investigate the mutual coherence among the generated sidebands. We first generate multiple AS sidebands by focusing Red $\left(\lambda_{R}=718 \mathrm{~nm}\right)$ and IR $\left(\lambda_{I R}=812 \mathrm{~nm}\right)$ beams into the $\mathrm{PbWO}_{4}$ crystal. Then a third (Yellow) beam is sent along the direction of the generated AS 3 sideband with a matching wavelength $\left(\lambda_{Y}=574 \mathrm{~nm}\right)$. Once the overlap in frequency, space, and time is achieved, the sidebands (AS 2 to AS 7) start to visibly flicker, due to interference between signals generated through different channels. We measure the pulse energy of AS 5 on a shot-by-shot basis by using a fast photodiode.

The statistics of the AS 5 pulse energy is shown in Fig. 11. Solid black bars give the histogram (number of pulses vs. AS 5 energy), with only Red and IR pulses applied at the input. This histogram shows a typical normal distribution, with about $10 \%$ average 
variations. However, with the addition of the Yellow beam at the input, the histogram of the AS 5 pulse energy (913 pulses total) transforms into a very different distribution (Fig. 11, white bars). We perform a simple calculation, which supports our qualitative understanding of this result. We consider interference of two fields (of the same frequency), whose intensities $\left(I_{1}\right.$ and $\left.I_{2}\right)$ fluctuate within $10 \%$ of their mean values. We further assume that the relative phase of these two fields varies randomly between 0 and $2 \pi$ (every value of $\Delta \varphi$ being equally probable). The resultant intensity $I=I_{1}+I_{2}+2 \sqrt{I_{1} I_{2}}[\cos (\Delta \varphi)]$ is expected to produce a histogram that is inversely proportional to the derivative of $I$ with respect to $\Delta \varphi$ and therefore has two peaks (at $I_{1}+I_{2}+2 \sqrt{I_{1} I_{2}}$ and $I_{1}+I_{2}-2 \sqrt{I_{1} I_{2}}$, where $\Delta \varphi$ equals 0 and $\pi$ respectively). This is exactly what our simulation shows (Fig. 11, dotted curve). In this simulation, we take the average value of $\left\langle I_{1}\right\rangle=0.41$ (arbitrary units) from the measurement, and find (from the best fit in Fig. 11) $<I_{2}>=0.09$. We repeat the calculation 91300 times (using random number generators), and divide the calculated number of counts (per intensity) by a factor of 100 when we compare the simulation with the experiment. The two peaks in the simulated histogram appear to be broadened by the fluctuations of $I_{1}$ and $I_{2}$, which are taken to be $10 \%$ each (matching the experimental observations). The peak on the right (at higher pulse energy) is calculated to be lower and broader than the peak on the left, in excellent qualitative agreement with the experimental data. This measurement, and its comparison with theory, confirms our expectation that the (highly-coherent) Raman process results in generation of mutually-coherent sidebands.

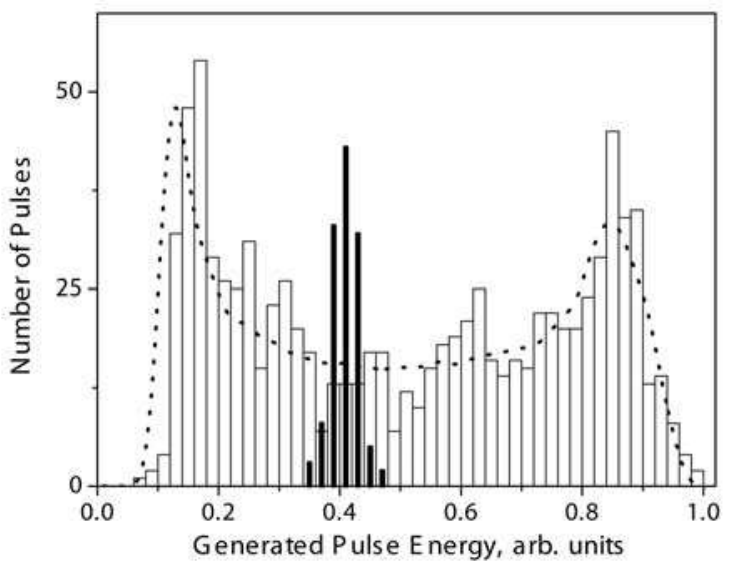

Fig. 11. Histograms of AS 5 pulse energy. Solid black bars: the number of pulses (out of 150) vs. AS 5 pulse energy generated with Red and IR input beams only. White bars: the histogram of AS 5 pulse energy (913 pulses total) with the addition of the third input beam. The dotted curve is a theoretical prediction obtained assuming perfect single-shot coherence of the two interfering fields, and random shot-to-shot variation of their relative phase.

\section{Discussion of broadband generation in Raman crystals with two-color laser fields}

In general, due to the large dispersion in solids, a non-collinear beam geometry is needed for effective excitation in a Raman crystal. In the CFWM method, the sample is so thin that 
the optical path is smaller than the Rayleigh range, the nonlinear length and the dispersion length. Therefore the linear dispersion and competing nonlinear optical effects such as SPM are minimized. In SRS and in Raman lasers high conversion efficiency is often achieved by using a much thicker sample or an external cavity. For example, when $\mathrm{PbWO}_{4}$ is used in a Raman laser, a typical thickness is up to $70 \mathrm{~mm}$ (Kaminskii et al., 2000). As a comparison, the sample we use is $1 \mathrm{~mm}$ thick, which is slightly thicker than the solid medium used in the CFWM method but is much thinner than the one used in the Raman laser.

The requirement of the frequency spacing between the two pump pulses to be tuned to Raman frequency is greatly relaxed since we are using fs pulses which have about $450 \mathrm{~cm}^{-1}$ bandwidth. Moreover, the wider Raman spectral linewidth (compared to gas medium) also eases the resonance requirement. We have observed Raman generation in diamond even when we tuned to double the Raman frequency. The best proof is that we observed the simultaneous excitation of $325 \mathrm{~cm}^{-1}$ and $901 \mathrm{~cm}^{-1}$, which leads to quantum beating in the CARS signal, as is shown in Fig. 16 later in the chapter.

A large number of adjustable experimental parameters offers increased flexibility, but at the same time requires careful alignment procedures in order to achieve day-to-day reproducibility. We normally start with the beam energy right below the SPM threshold. After we find the overlap and observe generation, we can increase the two pump beams power further without causing SPM. In a way, we can say that the Raman generation process suppresses the SPM process. A similar situation has been observed in the gas experiment using fs pulses (Sali et al., 2004). Both processes - the (Raman non-resonant) instantaneous FWM process and the Raman process often coexist. Each generated sideband is a result of the interplay between the two processes. The sidebands come out at almost equally separated angles but different peak frequency spacings. The intensity may not be monotonously decreasing as the order goes higher due to the large phase mismatch of certain sidebands. The spectral shapes of these lower-order sidebands sometimes show complex spectral shapes with multipeaks, either due to the simultaneous excitation of several Raman modes or the co-existence of the FWM process. The generated sidebands have similar pulse duration, close to those of the pump pulses (Liu \& Kobayashi, 2008).

In these experiments, we observe a larger number of AS sidebands than Stokes ones. This asymmetry is typically present since the Raman frequency is only one or two orders of magnitude smaller than the laser frequency, and hence the total generated bandwidth is comparable to the pump laser frequency. At substantially lower frequencies generation is intrinsically less efficient. In addition, infrared sidebands are harder to detect. Finally, in the present experiment phase matching occurs at a considerably larger output angle (and leads to a correspondingly worse spatial overlap with the pump beams) for a Stokes sideband compared to the same order AS sideband.

The sideband generation due to excitation of large- and small-frequency Raman modes shows few distinct differences. In the case of large-frequency Raman mode, a large angle is needed to fulfill the phasematching condition. As a result, the deviation of the frequency spacing from the Raman frequency is getting large as the sideband order goes higher. For broadband generation with $325 \mathrm{~cm}^{-1}$ or even lower frequency $191 \mathrm{~cm}^{-1}$, an almost constant frequency spacing can be maintained up to a very high order, therefore, more than 40 sidebands can be generated. Also, the spacing is close to the Raman frequency excited. The ideal case would be the case of using a gas when phase matching is fulfilled and many sidebands are generated (Chen et al., 2008). 
Compared to the fourier-transform-limited pulse excitation, temporal stretching of the excitation pulses allows us to use large pulse energies per unit area (while still avoiding parasitic nonlinear effects), and correspondingly increase the Raman coherence. Although the FWM process still creates some multiple peaks in the spectra of the low-order sidebands, the complications due to simultaneous excitation of multiple Raman lines are eliminated. Different Raman modes can be selectively excited by varying the time delay and the angle between the two pump beams.

At last, let's compare the generation between different types of crystals. Diamond has a higher damage threshold so more power can be used. With one single Raman mode, the sideband spectrum is cleaner. Due to the large Raman shift, the higher order sidebands lose phase matching quickly. Therefore, only around 20 sidebands are generated.

\section{Multicolor generation in Raman crystals with three-pulse excitation}

The generation in solids is complicated yet interesting. For example, broadband two dimensional (2-D) multicolored arrays with more than ten periodic columns and more than ten rows were generated in a sapphire plate using two crossed femtosecond laser beams overlapping in time and space has just been observed (Liu et al., 2009). Here we show two schemes with the additional third probe pulse irradiation on the sample. One scheme also leads to multicolor 2-D array generation. The three beams are arranged either in a planar (linear) configuration or Box CARS configuration (Demtröder, 2003) (see also Fig. 1 (c)).

\subsection{Multicolor generation in diamond using three-color laser fields}

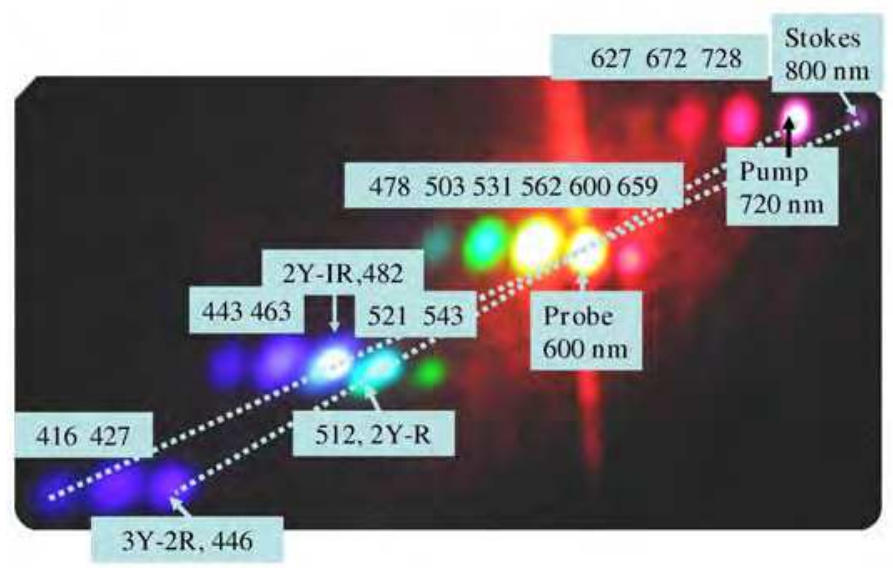

Fig. 12. The 2-D array generation in diamond with three input pulses $\left(\lambda_{\text {pump }}=720 \mathrm{~nm}, \lambda_{\text {Stokes }}=\right.$ $800 \mathrm{~nm}$, and $\lambda_{\text {probe }}=600 \mathrm{~nm}$ ). The wavelengths of the sidebands are labeled in $\mathrm{nm}$. The degenerated FWM signal (2Y-IR, a shorthand of $\left.2 \omega_{Y}-\omega_{I R}\right)$ from the probe and Stokes pulses and the one (2Y-R) from the probe and pump pulses are much stronger than the Raman generation spots. They either superimpose or shift slightly. The six-wave-mixing signal (3Y2R) from the pump and probe pulses is also visible.

When we apply a Stokes pulse (IR, $800 \mathrm{~nm}$ ), a pump pulse (red, $728 \mathrm{~nm}$ ) and a visible probe pulse (yellow, $600 \mathrm{~nm}$ ) at the usual boxed-CARS geometry, in addition to the strong CARS 
and coherent Stokes Raman scattering (CSRS) signals, we obtain a 2-D color array as shown in Fig. 12. The setup used here is similar to the one we used for visible and UV coherent Raman spectroscopy of dipicolinic acid and other biologically-relevant chemicals (Pestov et. al., 2005). The angle between the pump and Stokes beams is $5^{\circ}$. The probe beam is $11^{\circ}$ from the pump beam and $16^{\circ}$ from the Stokes beam. We propose the following explanation for the 2-D color array generation. The frequency spacing between the pump and Stokes pulses is around $1000 \mathrm{~cm}^{-1}$. As a result, the average sideband spacing is very regular, about 1040 $\mathrm{cm}^{-1}$. The sidebands in the first row are generated by the pump and Stokes pulses. The second row is generated by the CARS/CSRS and the high-order CARS processes. The FWM signal from the probe and Stokes/pump pulses leads to the generation of the third row. The degenerate FWM signal from the probe and Stokes pulses $\left(2 \omega_{Y}-\omega_{I R}\right)$ and from the probe and pump $\left(2 \omega_{Y}-\omega_{R}\right)$ pulses are much stronger than the generation spots due to Raman effect. They either superimpose or shift slightly. The six-wave-mixing signal $\left(3 \omega_{Y}-2 \omega_{R}\right)$ from the pump and probe pulses results in the fourth row sideband generation.

\subsection{Multicolor generation in $\mathrm{PbWO}_{4}$ using three-color laser fields}

We observe two-dimensional (2-D) multicolored Raman generation (Fig. 13 (a)) when we apply pump beam at $730 \mathrm{~nm}$, Stokes beam at $804 \mathrm{~nm}$, and probe beam at $604 \mathrm{~nm}$ in a Box CARS configuration. The angle between the pump, Stokes and probe beams are the same as those used in the diamond experiment, as described above. When we turn the crystal 90 degree so that the relative polarization to the crystal axis changes from $\mathrm{P}$ to $\mathrm{S}$, the 2-D multicolored Raman generation pattern changes dramatically and the sideband wavelength vary accordingly. The FWM signals produced by the three pulses sometimes overlap with the Raman sidebands, which leads to a generation of 2-D color array of similar nice round beams (Fig. 13 (a)). Although the FWM signal may overlap with one of the sidebands in space, the spectrum normally has two distinct peaks. When we increase the angle between the pump and Stokes input beams to 6 degrees while keeping the probe beam fixed, we observe up to 50 new generated colors (Fig. 13 (b)). We believe this is mainly due to the good phase matching between the pump beams and the resultant sidebands. Now the FWM signals are not overlapped with the Raman sidebands and appear as smaller beams (the smaller blue and green beams in between the regular 2-D color array).

We plot the measured peak frequencies of the Raman sidebands generated in the top 3 rows, the pump-Stokes row, the probe beam row and the bottom 4 rows as shown in Fig. 14 (a). The pump-Stokes row is marked by a dotted line in Fig. 13 (b). We find that the frequency spacing is close to the Raman frequency $901 \mathrm{~cm}^{-1}$. Therefore, it is clear that the sidebands are generated by the Raman processes. The frequency spacing between different rows is around $600 \mathrm{~cm}^{-1}$, which we believe is the result of phase matching of the probe pulse with the sideband beams generated by the pump and Stokes pulses.

We notice that the sidebands generated by pump and Stokes alone are strongly affected by the probe pulse. In Fig. 14 (b) we show the intensities of the first four AS with the probe pulse present and absent. We see that the addition of the probe pulse broadens the spectrum. We observe that the sidebands intensity is increased as well. When we change the probe delay so that the pulses don't overlap in time, the AS fields are not affected by the probe pulse. We plot the pump intensity before and after crystal in Fig. 14 (c). We conclude that more pump energy is converted to the sidebands when both Stokes and probe pulses are applied. 


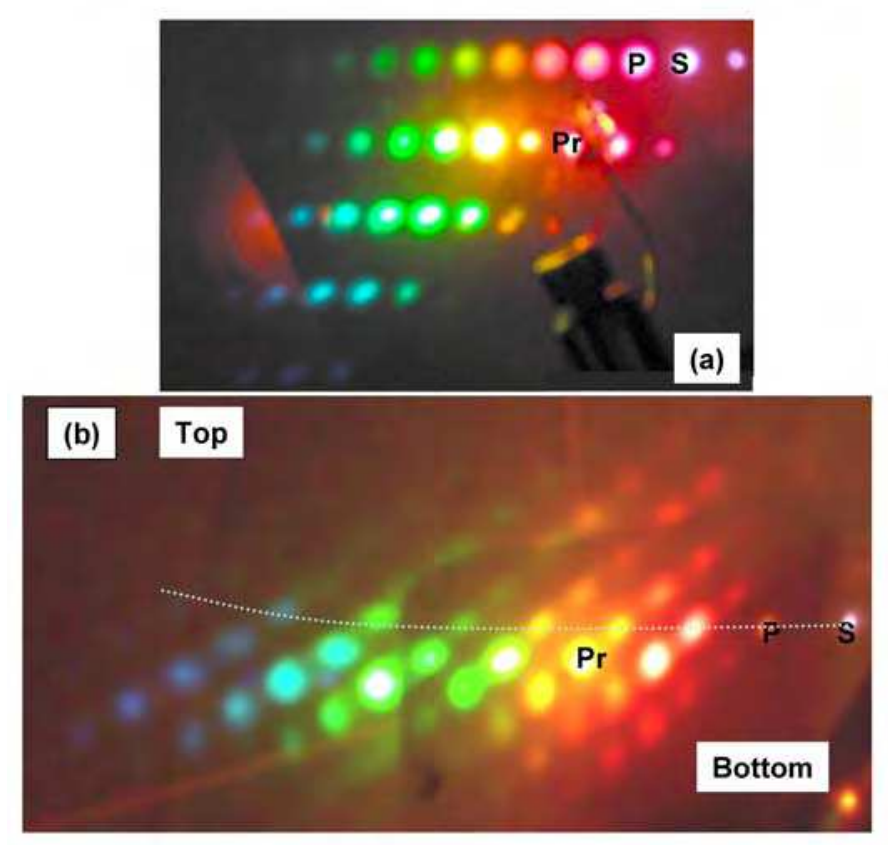

Fig. 13. (a) The Box CARS configuration. Three beams are sent in at the three corners of the box and the CARS signal is generated in the fourth corner. (b) and (c) Different 2-D patterns generated in $\mathrm{PbWO}_{4}$ under different input angles setting different phase matching conditions. In (b), a piece of small filter is used to block the strong probe beam for better picture. In (c), a large square neutral density filter is used to lower the intensity of the low order sidebands and three input beams. P: pump beam; S: Stokes beam; Pr: probe beam.

Next we use a planar configuration for the three input beams. We first generate sidebands using pump pulses at $755 \mathrm{~nm}$ and Stokes pulses at $803 \mathrm{~nm}$ as shown in the top of Fig. 15 (a). Then we send the third beam (which we also call probe beam) at $590 \mathrm{~nm}$ at the same direction and wavelength as AS 6. The configuration is shown in Fig. 15 (b). The probe beam and the pump beam satisfy the phase matching condition so that a strong FWM (green) signal is efficiently generated, which we believe acts as a seed to generate high-order sidebands (Fig. 15 (c)). We measure 18 sidebands with three pulses while with two pulses we generate 11 sidebands. The measured wavelength of the highest-order sideband is extended from $509 \mathrm{~nm}$ down to $467 \mathrm{~nm}$. Also the intensity of the high-order sidebands generated by two pulses is enhanced when a third probe beam is applied. We measure a $25 \%$ conversion efficiency from the probe beam to the sidebands. With two pulses, the high order sideband frequency spacing is $500 \mathrm{~cm}^{-1}$ per order (blue line in Fig. 15 (c)). It decreases to $350 \mathrm{~cm}^{-1}$ per order (cyan line in Fig. 15 (c)) when three pulses are used when more high order sidebands are generated. We would like to emphasize that this is a "proof of principle" study. A careful alignment and choice of the pump wavelengths should result in substantial additional improvement in sidebands generation. Because the probe beam doesn't have the exactly same wavelength as the AS 6, we have not observed the beating as described in our earlier paper (Zhi \& Sokolov, 2007). 

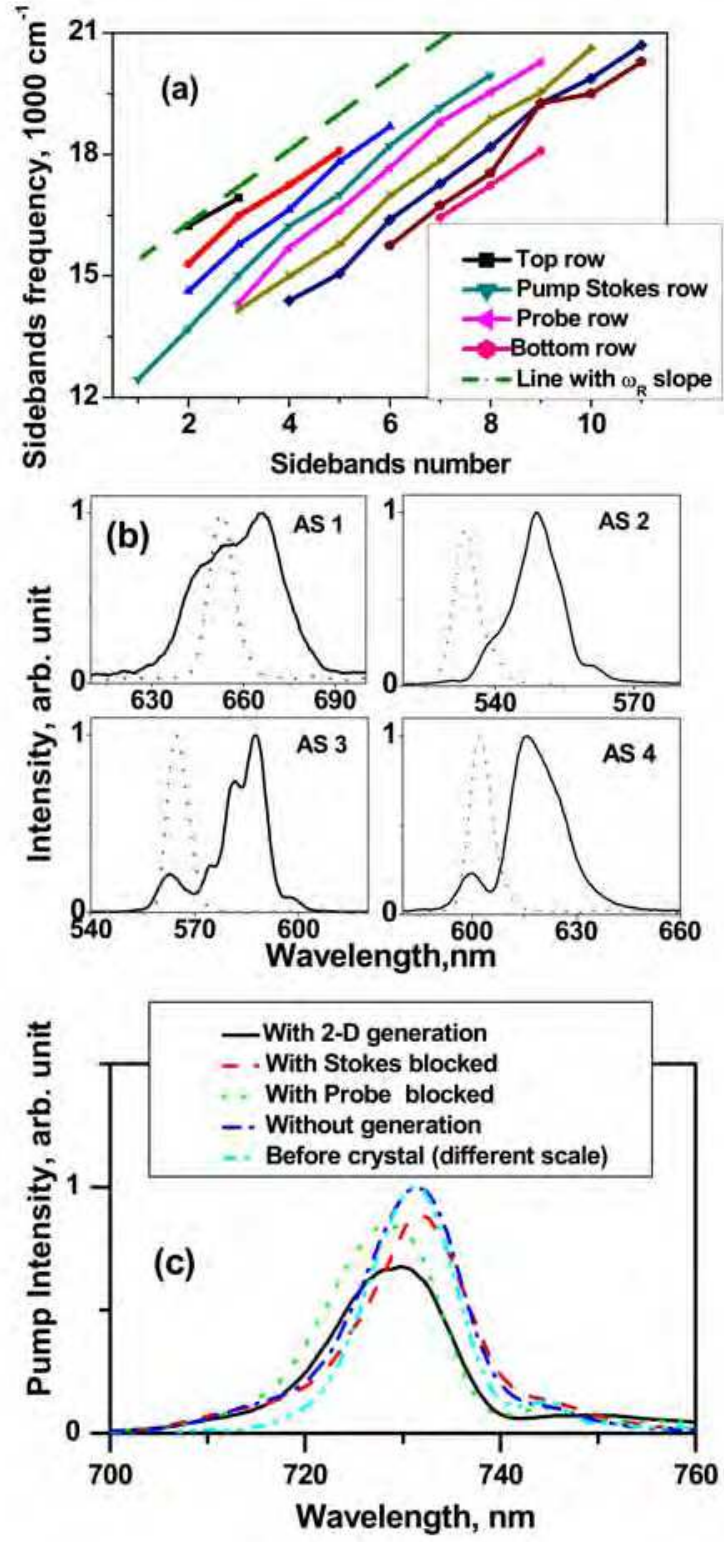

Fig. 14. Sidebands generation in $\mathrm{PbWO}_{4}$ with three pulses excitation in a Box CARS configuration. (a) Peak frequencies of the 2-D color array generation. The frequency is measured along or parallel to the pump-Stokes row (dotted line in Fig. 13 (c)). The dashed line has a slope of Raman frequency $901 \mathrm{~cm}^{-1}$. (b) solid line: AS sidebands generated by the three pulses; dashed line: AS sidebands generated by the pump and Stokes pulses only.

(c) Pump intensity with and without sideband generation. 


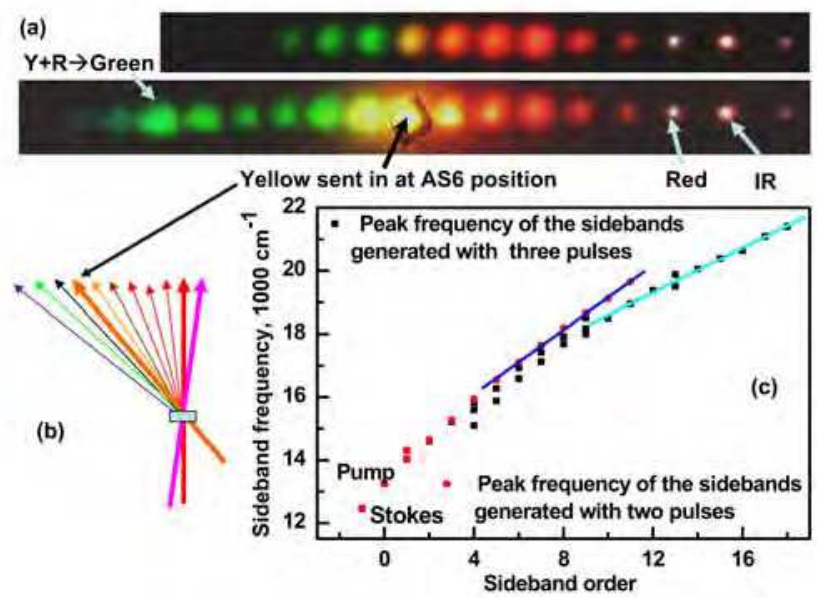

Fig. 15. Sidebands generation in $\mathrm{PbWO}_{4}$ with three pulses excitation in a planar configuration. The probe pulse is sent in at the same direction and wavelength as AS 6. (a) Generation pictures with pump and Stokes beams present (top picture) and all three pulses (pump, Stokes and probe) present (bottom). (b) Input beam geometry. (c) The sideband frequency as a function of the sideband order generated by two/three pulses.

\section{Coherence decay measurements in Raman crystals}

When a fs pulse are used as a probe, we observe the quantum beating between the two modes, which have a frequency difference of $565 \mathrm{~cm}^{-1}$. The corresponding beating period in the time domain is $59 \mathrm{fs}$, which is what we measure as shown in Fig. 16 (a). The CARS signal can be described by the following formula:

$$
\begin{aligned}
I_{C A R S} & =\left|A e^{-t / T_{2}}+B e^{i \Delta \omega t-t / T_{2}^{\prime}+i \varphi}\right|^{2} \\
& =A^{2} e^{-2 t / T_{2}}+B^{2} e^{-2 t / T_{2}^{\prime}} \\
& +2 A B e^{-t\left(1 / T_{2}+1 / T_{2}^{\prime}\right)} \cos (\Delta \omega t+\varphi) .
\end{aligned}
$$

By fitting the experimental data (Fig. 16 (b)) with the above equation, we get $T_{2}=1.3$ ps, $T_{2}^{\prime}=3.8 \mathrm{ps}$, and $\omega=106.5 \mathrm{rad} / \mathrm{ps}$, which is exactly the frequency difference between the two modes $\left(2 \pi / T=2 \pi *\left(3 / 100 * 565 \mathrm{~cm}^{-1}\right)=106.5\right)$.

When we use a narrow-band shaped probe beam (spectral width of about $1 \mathrm{~nm}$ ), we are able to measure the coherence decay of the two strong modes in the crystal as shown in Fig. 16 (c). Here the measured decay time for Raman mode at $328 \mathrm{~cm}^{-1}$ is 1.5 ps and 2.5 ps for 901 $\mathrm{cm}^{-1}$ mode. The discrepancy between our two measurements could be due to the finite pulse width.

We also measure the CARS decay time of the High Pressure High Temperature (HPHT) diamond sample that we used for our experiment. Using a pair of visible pulses as pump $(584 \mathrm{~nm})$ and Stokes $(635 \mathrm{~nm})$ and using a UV pulse (also about $50 \mathrm{fs}$ width) at $318 \mathrm{~nm}$ as a probe, we get the CARS signal at $305.8 \mathrm{~nm}\left(\delta v=1250 \mathrm{~cm}^{-1}\right)$ and CSRS signal at $332 \mathrm{~nm}$ $\left(\delta v=1326 \mathrm{~cm}^{-1}\right)$ as shown in Fig. 17. Undoubtedly, this is the strong Raman line in diamond. 


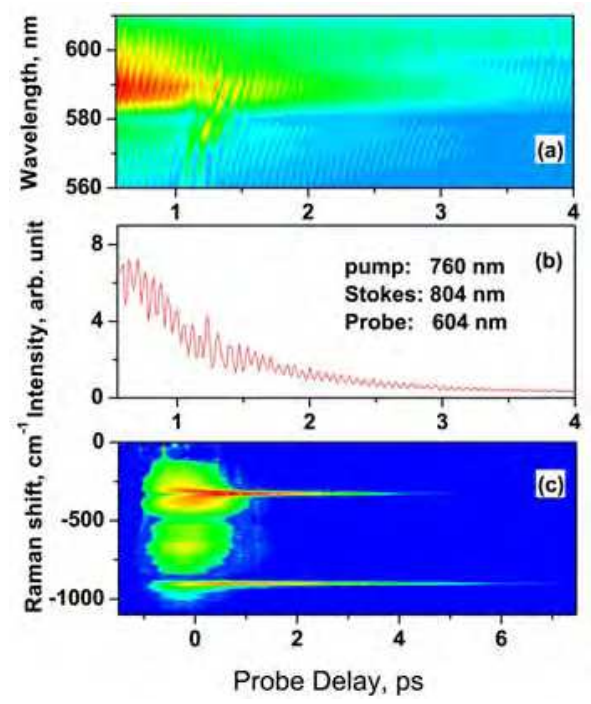

Fig. 16. (a) Quantum beating between the two strong Raman lines in $\mathrm{PbWO}_{4}$ measured by using a fs probe pulse with $\Delta v$ between pump and Stokes pulses equal to $720 \mathrm{~cm}^{-1}$. (b) The cross section from (a), which can be used to get the fitting parameters. The beating has a frequency of $106.5 \mathrm{rad} / \mathrm{ps}$, which corresponds to the Raman shift difference of $565 \mathrm{~cm}^{-1}$ between the two modes. (c)The coherence decay of the simultaneously excited strong Raman lines measured when fs pump, Stokes pulses and a narrow-band ps probe (about 1 nm spectral width) are applied to the crystal.
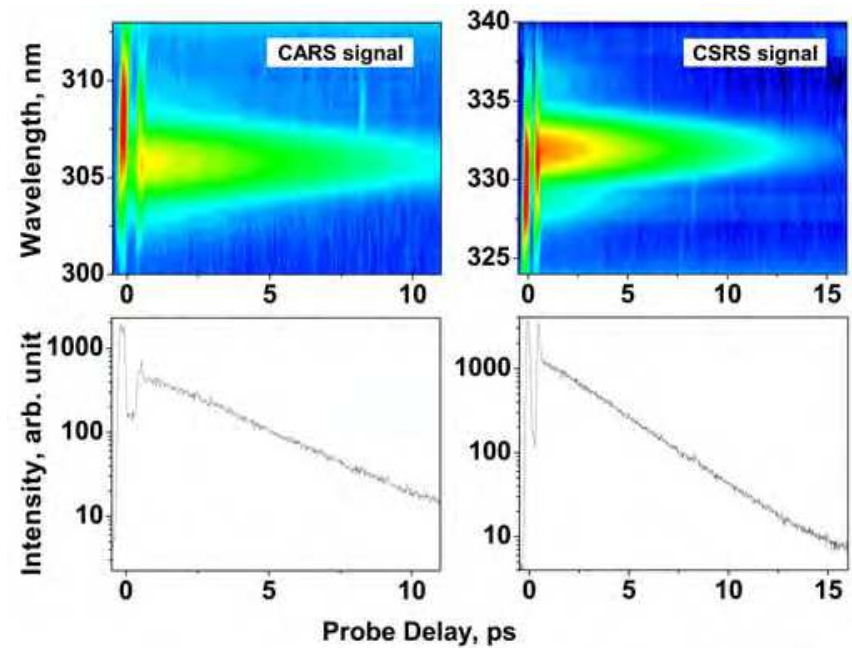

Fig. 17. The CARS and CSRS signals observed in diamond using a UV probe pulse. Top, the spectra of the CARS (left, center wavelength is $305 \mathrm{~nm}$ ) and CSRS (right, center wavelength is $332 \mathrm{~nm}$ ) as a function of the probe delay. Bottom, the exponential decay of the CARS (left) and CSRS (right) signals intensity average over their center wavelengths. Log scale is used. 
The width of the line in the SRS spectrum related to SRS-promoting vibration transition is $\Delta \nu_{R} \approx 2.5 \mathrm{~cm}^{-1}$. Therefore the phonon relaxation time $T_{2}=\left(\pi c \Delta \nu_{R}\right)^{-1} \approx 4.2$ ps (Kaminskii et al., 2005). We measure the exponential decay of the CARS/CSRS signal as shown in Fig. 17 (bottom). We get the intensity of the CARS/CSRS signal by averaging over the center wavelengths. To remove the effect of the strong instantaneous FWM signal over the CARS/CSRS signal, we only fit the data about 2 ps after the 0 delay with an exponential decay and obtain decay time $T_{2}$ as 2.6 and 2.7 ps for CARS and CSRS, respectively. There is a gap between the FWM and the CARS signal, which remains a mystery to us. Similar gap has also been observed in $\mathrm{PbWO}_{4}$ crystal.

We observe strong absorption of the UV beam when it is used as a probe while a pair of visible pulses are used for excitation. The absorption only happens when the pulses overlap in time. The exact origin of this absorption is unknown, but most likely it is related to a twophoton electronic excitation process. We identify that the width of the absorption dip is related to the group dispersion delay of the different pulses.

\section{Conclusion and future work}

We realize efficient generation of light frequencies spanning the infrared, visible, and ultraviolet spectral regions in Raman crystals, which provides a possible new way to generate record-short (subfemtosecond) pulses. Our experimental work lends insight to the complicated sideband generation process in crystals. From working with the popular Raman crystal $\mathrm{PbWO}_{4}$, we find that first of all, it is very important to satisfy the phase matching condition, i.e. non-collinear geometry has to be used for efficient generation in the crystal. By varying the detuning, angles, and polarization of the pump and Stokes beams, we find that FWM and Raman effects coexist and the generated sideband frequencies are very sensitive to the angles and the polarization of the pump beams. We design an interference experiment which proves the mutual coherence among the Raman sidebands generated. When a third probe beam is applied in the same plane with the pump and Stokes pulses, more sidebands are generated and the energy of the high-order sidebands is enhanced. When the three laser fields (pump, Stokes and probe) are applied to the crystal in a Box CARS configuration, we observe that up to 50 colors are generated which form a 2-D color array.

We demonstrate similar generation in another crystal, diamond, which shows that the Raman generation method is universal and can be applied to other popular Raman crystals. Since diamond is an isotropic material with a simpler dispersion relationship compared to that of the uniaxial crystal $\mathrm{PbWO}_{4}$, the theoretical phase-matching calculation can easily be performed and can help understand the sideband generation process. The experiment done in diamond also sheds some light on how the 2-D color array is generated when three pulses are applied to the diamond crystal.

Compared to the Raman generation using fs fourier transform limited pulses, we obtain more efficient sidebands generation in $\mathrm{PbWO}_{4}$ crystal with more sidebands generated by using a pair of time-delayed linearly chirped pulses. Up to 40 AS and $5 \mathrm{~S}$ are observed. As high as $41 \%$ of pump and $21 \%$ of Stokes conversion efficiency is measured. This shows that this is a good way to build maximal coherence in the crystal. The pulses have ps duration, which is on the order of the coherence decay time. This means ps pulses may be more effective for sideband generation than fourier transform limited fs pulses.

Many other future projects can be extended from this research. Since this is a very new and challenging experiment, there are many "mysteries" remaining to be solved. For example, the splitting in the time domain of the FWM and CARS signal, the exact mechanism of 2- D color array generation when three pulses are used, and the UV absorption when pulses are 
overlapped. The unequal frequency spacing between the sidebands needs more vigorous theoretical examination. It would be helpful if we could calculate and predict how many sidebands and at what angles are generated. It would not only help us understand how the phase matching condition, Raman generation, FWM interplay with each other, it could also help us find the best condition for efficient sidebands generation. Experimentally, one will learn more of the sideband generation in crystals when more quantitative results are obtained. For example, it will be important to characterize the sidebands, i.e. to measure the absolute sideband pulse energy and the sideband pulse width. The characterization also can help us find the optimal experimental condition for sidebands generation. For example, one can study how the generation efficiency varies as a function of the relative delay between the two pulses. If the sidebands generated in the crystal are chirped when linearly chirped input pulses are used, we might be able to compress the pulses later to get shorter pulses, as has been demonstrated (Liu \& Kobayashi, 2009).

In the near future, we envision driving one of these Raman transitions by a pair of timedelayed chirped picosecond pulses, and then using another (compressed or shaped) fs pulse from the same source laser, to probe the Raman coherence prepared by the two excitation pulses. Our experiment has already shown efficient spectral broadening of a (third) probe pulse; future experiments will possibly lead to substantial pulse compression in the same medium, as predicted in our earlier work (Sokolov \& Harris, 2003; Sokolov et al., 2005).

Whether the collinear generation in $\mathrm{PbWO}_{4}$ crystal by the excitation of the small-frequency Raman mode at $325 \mathrm{~cm}^{-1}$ can be realized or not deserves to be studied, since collinear generation will result in the convenience of combining the sidebands for sub-fs pulse synthesis. Using two separately prepared linearly chirped pulses for the excitation of the Raman mode at $903 \mathrm{~cm}^{-1}$ in $\mathrm{PbWO}_{4}$ crystal is also a promising experiment given the efficient sidebands generation from the excitation of the Raman mode with a lower frequency. An alternative way of excitation would be to use a pulse shaper to obtain a pulse train with a period equals to the period of Raman vibrations. If ps pulses are available, a comparison of sideband generation using ps pulses, fs pulses, and linearly chirped pulses would be an interesting subject as well. Optimizing the sideband generation in diamond is possible if a good sample with the right thickness is available. We have observed Stokes generated in the IR region, where diamond has a very small dispersion. Therefore it is a promising candidate material for far IR generation given its wide transmission spectral range. Broadband light generation in other types of crystals such as $\mathrm{KGW}\left(\mathrm{KGd}\left(\mathrm{WO}_{4}\right)_{2}\right.$, a very popular Raman crystal due to its high efficiency) and CVD diamond (both polycrystalline and single crystal form) will be studied next. CVD diamond is much cheaper than the natural one, and high quality samples are available now. The high damage threshold and strong single Raman line are very desirable properties for our purpose.

The important future project will be to realize the goal of the generation of sub-fs pulses using the broadband light generated in crystals. First of all we can try to realize the collinear generation in the crystal by exciting the lower Raman frequency in $\mathrm{PbWO}_{4}$. Then we can use a pulse shaper, such as Dazzler (FASTLITE) to combine the sidebands generated by the chirped pulse excitation. For the angled output, one possible scheme is combine the generated sidebands using a 4-f grating setup, as has been demonstrated recently (Matsubara et al., 2008; Weigand et al., 2009).

\section{Acknowledgement}

We acknowledge support from National Science Foundation (Grants no. PHY354897 and 722800), Texas Advanced Research Program (Grant no. 010366-0001-2007), the Army 
Research Office (Grant no. W911NF-07-1-0475), and the Robert A. Welch Foundation (Grant no. A-1547).

\section{References}

Basiev, T. T., Sobol, A. A., Zverev, P. G., Osiko, V. V. \& Powell, R. C. (1999). Comparative Spontaneous Raman Spectroscopy of Crystals for Raman Lasers, Applied Optics Vol. (38): 594-598.

Basiev, T. T., \& Powell, R. C. (1999). Introduction, Optical Materials Vol. (11): 301-306.

Basiev, T. T., Sobol, A. A., Voronko, Y. K. \& Zverev, P. G. (2000). Spontaneous Raman spectroscopy of tungstate and molybdate crystals for Raman lasers, Optical Materials Vol. (15): 205-216.

Basiev, T. T., Osiko, V. V., Prokhorov, A. M. \& Dianov, E. M. (2003). Crystalline and Fiber Raman Lasers, in Solid-State Mid-Infrared Laser Sources, Springer, Berlin / Heidelberg, pp. 359-408.

Basiev, T. T., Zverev, P. G., Karasik, A. Y., Osiko, V. V., Sobol, A. A. \& Chunaev, D. S. (2004). Picosecond stimulated Raman scattering in crystals, Journal of Experimental and Theoretical Physics Vol. (99): 934-941.

Chen, W., Hsieh, Z., Huang, S. W., Su, H., Lai, C., Tang, T., Lin, C.,Lee, C., Pan, R., Pan, C. \& Kung, A. H. (2008). Sub-Single-Cycle Optical Pulse Train with Constant Carrier Envelope Phase, Phys. Rev. Lett. Vol. (100): 163906.

Crespo, H., Mendonça, J. T. \& Dos Santos, A. (2000). Cascaded highly nondegenerate fourwave-mixing phenomenon in transparent isotropic condensed media, Opt. Lett. Vol. (25): 829-831.

Demtröder, W. (2003). Laser spectroscopy: Basic concepts and Instrumentation, 3rd Edition, Springer, New York.

Dudovich, N. Oron, D. \& Silberberg, Y. (2002). Single-pulse coherently controlled nonlinear Raman spectroscopy and microscopy, Nature Vol. (418): 512-514.

Dyson, A., Dangor, A. E., \& Dymoke-Bradshaw, A. K. L. (1989). Sideband generation by coherent anti-Raman scattering in quartz of a two-frequency high-power laser beam, Journal of Physics B: Atomic, Molecular and Optical Physics Vol. (22): L231-L233.

Eckhardt, G., Bortfeld D. P. \& Geller, M. (1963). Stimulated Emission of Stokes and AntiStokes Raman Lines from Diamond, Calcite, and Alpha-sulfur Single Crystals, Appl. Phys. Lett. Vol. (3): 137-138.

Edwards, D. F. \& Ochoa, E. (1981). Infrared refractive index of diamond, J. Opt. Soc. Am. Vol. (71): 607-608.

Schulz-Von der Gathen, V., Bornemann, T., Kornas, V. \& Dobele, H. F. (1990). VUV generation by high-order CARS, Quantum Electronics, IEEE Journal of Vol. (26): 739743.

Gershgoren, E., Bartels, R. A., Fourkas, J. T., Tobey, R., Murnane, M. M. \& Kapteyn, H. C. (2003). Simplified setup for high-resolution spectroscopy that uses ultrashort pulses, Opt. Lett. Vol. (28): 361-363.

Imasaka, T., Kawasaki, S., \& Ishibashi, N. (1989). Generation of more than 40 laser emission lines from the ultraviolet to the visible regions by two-color stimulated raman effect, Applied Physics B: Lasers and Optics Vol. (49): 389-392.

Inoue, K., Kato, J., Hanamura, E., Matsuki H., \& Matsubara, E. (2007). Broadband coherent radiation based on peculiar multiple Raman scattering by laser-induced phonon gratings in $\mathrm{T}_{i} \mathrm{O}_{2}$, Phys. Rev. B Vol. (76): 041101(R). 
Kaminskii, A. A., McCray, C. L., Lee, H. R., Lee, S. W., Temple, D. A., Chyba, T. H., Marsh, W. D., Barnes, J. C., Annanenkov, A. N., Legun, V. D., Eichler, H. J., Gad, G. M. A. \& Ueda K. (2000). High efficiency nanosecond Raman lasers based on tetragonal $\mathrm{PbWO}_{4}$ crystals, Optics Communications Vol. (183): 277-287.

Kaminskii, A. A., Ralchenko V. G., Konov, V. I. \& Eichler, H. J. (2005). High-order Stokes and anti-Stokes Raman generation in CVD diamond, Physica Status Solidi (B) Vol. (242): R4-R6.

Kaminskii, A. A., Ralchenko V. G. \& Konov, V. I. (2006). CVD-diamond - a novel $\chi^{3-}$ nonlinear active crystalline material for SRS generation in very wide spectral range, Laser Physics Letters Vol. (3): 171-177.

Katzman, S. (2001). Rainbow Stars: A spectrum of possibilities, American Chemical Society magazine News in Brief Vol. (73): 351.

Kawano, H., Lin, C. H. \& Imasaka, T. (1996). Generation of high-order rotational lines by four-wave Raman mixing using a high-power picosecond Ti:Sapphire laser, Applied Physics B: Lasers and Optics Vol. (63):121-124.

Kawano, H., Hirakawa, Y., \& Totaro, I. (1997). Generation of more than 40 rotational Raman lines by picosecond and femtosecond Ti:sapphire laser for Fourier synthesis, Applied Physics B: Lasers and Optics Vol. (65): 1-4.

Kawano, H., Hirakawa, Y. \& Imasaka, T. (1998). Generation of high-order rotational lines in hydrogen by four-wave Raman mixing in the femtosecond regime, Quantum Electronics, IEEE Journal of Vol. (34): 260-268.

Kawano, H., Mori, T., Hirakawa, Y., \& Imasaka, T. (1999). Use of phase-locking in four-wave Raman mixing for generating ultrashort optical pulses, Physical Review A Vol. (59): 4703-4715.

Kienberger, R., Goulielmakis, E., Uiberacker, M., Baltuska, A., Yakovlev, V., Bammer, F., Scrinzi, A., Westerwalbesloh, Th., Kleineberg, U., Heinzmann, U., Drescher M., \& Krausz, F. (2004) Atomic transient recorder, Nature Vol. (427): 817-821.

Korn, G., Dühr, O., \& Nazarkin, A. (1998). Observation of Raman Self-Conversion of fs-Pulse Frequency due to Impulsive Excitation of Molecular Vibrations, Physical Review Letters Vol. (81): 1215-1218.

Liang, J. Q., Katsuragawa, M., Kien, F. L. \& Hakuta, K. (2000). Sideband Generation Using Strongly Driven Raman Coherence in Solid Hydrogen, Phys. Rev. Lett. Vol. (85): 2474- 2477.

Liu, J. \& Kobayashi, T. (2008). Cascaded four-wave mixing and multicolored arrays generation in a sapphire plate by using two crossing beams of femtosecond laser, Opt. Express Vol. (16): 22119-22125.

Liu, J. \& Kobayashi, T. (2009). Generation of sub-20-fs multicolor laser pulses using cascaded four-wave mixing with chirped incident pulses, Optics Letters Vol. (34): 24022404.

Liu, J., Kobayashi, T. \& Wang, Z. (2009). Generation of broadband two-dimensional multicolored arrays in a sapphire plate, Opt. Express Vol. (17): 9226-9234.

Matsubara, E., Inoue K. \& Hanamura, E. (2006). Dynamical symmetry breaking induced by ultrashort laser pulses in $\mathrm{KTaO}_{3}$, J. Phys. Soc. Jpn. Vol. (75): 024712 .

Matsubara, E., Sekikawa, T. \& Yamashita, M. (2008). Generation of ultrashort optical pulses using multiple coherent anti-Stokes Raman scattering in a crystal at room temperature, Appl. Phys. Lett. Vol. (92): 071104.

Matsubara, E., Kawamoto, Y., Sekikawa, T. \& Yamashita, M. (2009). Generation of ultrashort optical pulses in the10 fs regime using multicolor Raman sidebands in $\mathrm{KTaO}_{3}$, Opt. Lett. Vol. (34): 1837-1839. 
Matsuki, H., Inoue, K. \& Hanamura, E. (2007). Multiple coherent anti-Stokes Raman scattering due to phonon grating in $\mathrm{KNbO}_{3}$ induced by crossed beams of two-color femtosecond pulses Phys. Rev. B Vol. (75): 024102.

Nazarkin, A., Korn, G., Wittmann, M., \& Elsaesser, T. (1999). Generation of Multiple PhaseLocked Stokes and Anti-Stokes Components in an Impulsively Excited Raman Medium, Phys. Rev. Lett. Vol. (83): 2560-2563.

Pask, H. M. (2003). The design and operation of solid-state Raman lasers, Progress in Quantum Electronics Vol. (27): 3-56.

Pestov, D., Zhi, M., Sariyanni, Z. E., Kalugin, N. G., Kolomenskii, A. A., Murawski, R. K., Paulus, G. G., Sautenkov, V. A., Schuessler, H., Sokolov, A. V., Welch, G. R., Rostovtsev, Y. V., Siebert, T., Akimov, D. A., Graefe, S., Kiefer, W. \& Scully, M. O. (2005). Visible and UV coherent Raman spectroscopy of dipicolinic acid, Proc. Nat. Acad. Sci. U. S. A. Vol. (102): 14976-14981.

Pierson, H. O. (1993). Handbook of Carbon Graphite, Diamond and Fullerenes: Properties, Processing, and Applications, Noyes Publications.

Sali, E., Mendham, K. J., Tisch, J. W. G., Halfmann, T. \& Marangos, J. P. (2004). High-order stimulated Raman scattering in a highly transient regime driven by a pair of ultrashort pulses, Opt. Lett. Vol. (29): 495-497.

Sali, E., Kinsler, P., New, G. H. C., Mendham, K. J., Halfmann, T., Tisch, J. W. G. \& Marangos, J. P. (2005). Behavior of high-order stimulated Raman scattering in a highly transient regime, Physical Review A Vol. (72): 013813.

Sokolov, A. V. \& Harris, S. E. (2003). Ultrashort pulse generation by molecular modulation, Journal of Optics B: Quantum and Semiclassical Optics Vol. (5): R1-R26.

Sokolov, A. V., Shverdin, M. Y., Walker, D. R., Yavuz, D. D., Burzo, A. M. Yin, G. Y. \& Harris, S. E. (2005). Generation and control of femtosecond pulses by molecular modulation, Journal of Modern Optics Vol. (52): 285-304.

Smith, D. Y., Inokuti M. \& Karstens, W. (2001). A generalized Cauchy dispersion formula and the refractivity of elemental semiconductors, J. Phys.: Condens. Matter Vol. (13): 3883- 3893.

Steinmeyer, G., Sutter, D. H., Gallmann, L., Matuschek N. \& Keller U., (1999). Frontiers in Ultrashort Pulse Generation: Pushing the Limits in Linear and Nonlinear Optics, Science, Vol. (286): 1507-1512.

Takahashi, J. (2004), Generatoin of a broadband spectral comb with multiwave mixing by exchange of an impulsively stimulated phonon, Opt. Express Vol. (12): 1185-1191.

Weigand, R., Mendonca, J. T. \& Crespo, H. M. (2009). Cascaded nondegenerate fourwavemixing technique for high-power single-cycle pulse synthesis in the visible and ultraviolet ranges, Phys. Rev. A Vol. (79): 063838.

Yoshikawa, S. \& Imasaka, T.(1993). A new approach for the generation of ultrashort optical pulses, Optics Communications Vol. (96): 94-98.

Zheltikov, A. M. (2002). Spectroscopic and quantum-control aspects of ultrashort-pulse synthesis through impulsive high-order stimulated Raman scattering, Journal of Raman Spectroscopy Vol.(33): 112-120.

Zhi, M. \& Sokolov, A. V. (2007). Broadband coherent light generation in a Raman-active crystal driven by two-color femtosecond laser pulses, Opt. Lett. Vol.( 32): 2251-2253.

Zhi, M. \& Sokolov, A. V. (2008). Broadband generation in a Raman crystal driven by a pair of time-delayed linearly chirped pulses, New J. Phys. Vol.(10): 025032.

Zhi, M., Wang, X. \& Sokolov, A. V. (2008). Broadband coherent light generation in diamond driven by femtosecond pulses, Opt. Express Vol.(16): 12139-12147. 


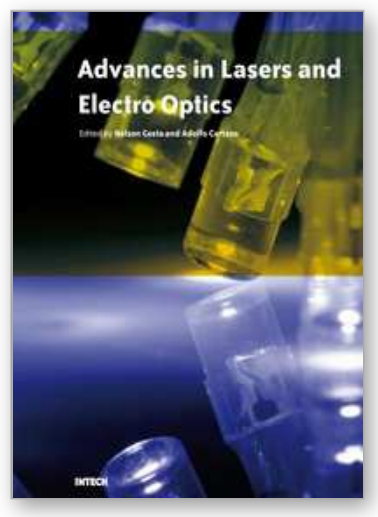

\author{
Advances in Lasers and Electro Optics \\ Edited by Nelson Costa and Adolfo Cartaxo
}

ISBN 978-953-307-088-9

Hard cover, 838 pages

Publisher InTech

Published online 01, April, 2010

Published in print edition April, 2010

Lasers and electro-optics is a field of research leading to constant breakthroughs. Indeed, tremendous advances have occurred in optical components and systems since the invention of laser in the late $50 \mathrm{~s}$, with applications in almost every imaginable field of science including control, astronomy, medicine, communications, measurements, etc. If we focus on lasers, for example, we find applications in quite different areas. We find lasers, for instance, in industry, emitting power level of several tens of kilowatts for welding and cutting; in medical applications, emitting power levels from few milliwatt to tens of Watt for various types of surgeries; and in optical fibre telecommunication systems, emitting power levels of the order of one milliwatt. This book is divided in four sections. The book presents several physical effects and properties of materials used in lasers and electro-optics in the first chapter and, in the three remaining chapters, applications of lasers and electro-optics in three different areas are presented.

\title{
How to reference
}

In order to correctly reference this scholarly work, feel free to copy and paste the following:

Miaochan Zhi, Xi Wang and Alexei V. Sokolov (2010). Broadband Light Generation in Raman-active Crystals Driven by Femtosecond Laser Fields, Advances in Lasers and Electro Optics, Nelson Costa and Adolfo Cartaxo (Ed.), ISBN: 978-953-307-088-9, InTech, Available from: http://www.intechopen.com/books/advancesin-lasers-and-electro-optics/broadband-light-generation-in-raman-active-crystals-driven-by-femtosecond-laserfields

\section{INTECH}

open science | open minds

\section{InTech Europe}

University Campus STeP Ri

Slavka Krautzeka 83/A

51000 Rijeka, Croatia

Phone: +385 (51) 770447

Fax: +385 (51) 686166

www.intechopen.com

\section{InTech China}

Unit 405, Office Block, Hotel Equatorial Shanghai

No.65, Yan An Road (West), Shanghai, 200040, China

中国上海市延安西路65号上海国际贵都大饭店办公楼405单元

Phone: +86-21-62489820

Fax: $+86-21-62489821$ 
(C) 2010 The Author(s). Licensee IntechOpen. This chapter is distributed under the terms of the Creative Commons Attribution-NonCommercialShareAlike-3.0 License, which permits use, distribution and reproduction for non-commercial purposes, provided the original is properly cited and derivative works building on this content are distributed under the same license. 